\section{PRODUCT DESIGN CONCEPT EVALUATION BY USING ANALYTICAL HIERARCHY AND ANALYTICAL NETWORK PROCESSES}

\author{
Hüseyin Özkal ÖZSOY*, Çiğdem YILMAZ ÖZSOY**
}

Received: 28.11.2015; Final Text: 24.05.2018

Keywords: Analytical hierarchy process; analytic network process; design concept selection; product design; hand cart design.

\section{INTRODUCTION}

Ulrich and Eppinger (2008) define the design process in six steps: 1. Investigating user needs, 2. Concept design, 3. Initial development, 4. Concept evaluation and detailed design of the selected concept, 5. Design checks by drawings, models and mock-ups, 6 . Information exchange with engineers and customers. Concept design at Step 2 is an important part of the design process in which design solutions are found and product functions are structured prior to the detail design processes which would require great investment and create heavy workload.

For concept design selection, designers evaluate a number of design concept ideas according to criteria such as their creative thoughts, user needs and known specs of successful products and then decide on which one of the available ideas should be selected for further improvement (Xiao et al., 2007). When the design concept is selected, the main structure and form of the product that is the main target to achieve will be strictly known (Kim and Lee, 2010). Therefore this task directly affects the success of both the design process and the final product (Salonen and Perttula, 2005; Ayağ, 2005). Correctly deciding on which design concept to continue working on is important as an incorrect selection may cause problems that might be difficult to resolve later (Hsu and Woon, 1998; Zhang et al., 2006). A good selection at this phase means good sale, customer satisfaction and high profits as well as shorter development time and cost. Mistakes made during design concept selection might increase the cost and the duration of the design process due to repetitive corrections. Eventually this may lead to the targeted product design not being completed within the available time-budget and may even cause a total project failure. Therefore, a proper concept selection, performed at the early stages of the product design process is important for product success (Fung et al., 2007).

Concept design selection involves complex multi-criteria decision making tasks that can be simplified by using suitable tools (Xu et al., 2007).

\footnotetext{
* Department of Architecture, Faculty of Architecture, Mimar Sinan Fine Arts University, Istanbul, Turkey.

** Department of Econometrics, Faculty of Economics, Istanbul University, Istanbul, Turkey.
} 
Different evaluation trends and tools are available for this purpose such as Simulation, TOPSIS, GIS, Goal Programming, DEA, Delphi, balanced scorecard, factor analysis, fuzzy logic model, genetic algorithm, SWOT analysis, AHP and ANP (Sipahi and Timor, 2010).

Contemporary decision making processes in industrial design are user-centered, ethnographic, collective and participatory (Nova 2014). Participatory or cooperative design consists of a distinct set of design and research practices, in which designers and users actively work together for improving the quality of life (Halskov and Hansen, 2015). This article investigates the Analytical Hierarchy Process (AHP) and the Analytical Network Process (ANP) methods and then proposes a model to use them in the product design process to evaluate industrial design concepts. The reasons behind the selection of AHP and ANP as the research methods were their participatory structure and ability to process subjective data gathered from user interviews, enabling the users to be placed at the center of the design process. The reasons for applying these methods in the industrial design process are for increasing the accuracy of decision making, simplifying the overall work by using a methodic approach and making the process more user-centered.

This section briefly covered the product design and design concept selection processes. In the following sections, the uses of the AHP and ANP methods in the literature are reviewed. Then the methods are described and their adaptation to the industrial design process as design concept selection methods are explained. Finally, their application is demonstrated with a study in which seven selected design concepts are evaluated by using both methods and the one having the highest performance score is selected for further design development.

\section{LITERATURE REVIEW}

A search of the academic literature brings up a substantial amount of studies from different fields that utilize AHP and ANP in various specific ways and for different purposes. Sipahi and Timor (2010) performed a study to produce a general overview of AHP and ANP applications by investigating over 600 research papers. Their study gives good overall information about the literature pointing out that the methods are increasingly being used in various scientific fields ranging from manufacturing, environmental management, agriculture, energy management, transportation, construction, healthcare to education, logistics, e-business, information technologies, research and development, telecommunications, finance, military, government, marketing, tourism, archeology, auditing and mining.

In our own research of the literature concentrated on the use of AHP and ANP mainly on design related issues, we saw that the methods are used in several main ways; a group of studies singlehandedly use either AHP or ANP such as the following. Battistoni et al. (2013) used AHP to investigate the user response to the products being developed. Hambali et al. (2008) worked on using AHP in concept design selection tasks of the design process. In their study, they used AHP to evaluate disability chair design concepts to select one of them for production. Hambali et al. (2009) used AHP to determine the production method for a product concept. They demonstrated the use of their method in an application in which they evaluated production methods for a composite car bumper. Sarfaraz 
and Jenab (2012) proposed a model based on fuzzy AHP to be used in concept design evaluation. They demonstrated the use of this model in an applied study in which several hospital bed design concepts are evaluated. Boonkanit and Aphikajornsin (2009) studied the use of ANP on the evaluation of ecological product design concepts.

Some studies incorporate AHP and ANP together cooperatively and comparatively such as the following. Cheng and Li (2004) used AHP and ANP to propose a method for evaluation-selection of industrial production firms and compared the two methods in terms of their applications. Sharma et al. (2015) proposed a method which was based on AHP and ANP to select the most suitable material for the production of a product component. Eshtehardian et al. (2012) proposed a method based on ANP and AHP to evaluate material providers. Begičević et al. (2007) made a research in which they used the AHP and ANP methods comparatively on strategic planning. Graham (2012) proposed a hybrid AHP-ANP methodology for the evaluation and selection of sustainable transportation networks. Azizi and Maleki (2014) comparatively used the AHP and ANP methods for evaluation of the provider firms in automotive industry. Beltrán et al. (2014) proposed a multi criteria decision making approach based on AHP-ANP for investigating the profitability of investments.

Other studies use AHP and ANP together with additional methods such as the following. Bedessi and Lisi (2011) proposed a hybrid method in which the AHP, ANP and ANN (Artificial Neural Networks) methods were used together for multi criteria decision making problems and they investigated the advantages and disadvantages of their model, pointing out the technical differences of the three processes. Marini et al. (2016) worked on ecological product design and investigated the use of AHP and ANP together with Quality Function Deployment (QFD) in concept design and material selection for making better decisions to improve product success. Gupta et al. (2015) proposed a method based on TOPSIS and fuzzy AHP which can be used in concept design evaluation. They demonstrated their method in an application in which various suitcase design concepts were evaluated according to criteria like cost, quality, human and environmental factors. Renzi et al. (2017) researched the use of AHP and ANP together with other multi-criteria decision methods for design evaluation in the automotive industry, trying to transfer knowledge on decision making methods to the industrial context. Toksari and Toksari (2011) used fuzzy AHP to evaluate strategies for evaluating target markets of white goods. Nagahanumaiah et al. (2007) developed a method in which Fuzzy AHP and Quality Function Deployment (QFD) are used together in visual C++ programming language for evaluation and selection of rapid production tools. They also compared traditional manufacturing methods with today's rapid production methods according to cost. Hsiao (2002) used AHP together with QFD, Failure Mode Effects Analysis (FMEA) and Design for Assembly (DFA) to introduce a process to help designing competitive toys suitable for infants. Kwong and Bai (2003) used AHP and triangular fuzzy numbers to improve hair drier designs by calculating weighted importance values of user needs. Felice and Petrillo (2010) used AHP and QFD together in a study to gather data about the needs of ceramic product users. Delice and Güngör (2009) performed a user-centered research to analyze the usability problems of website designs by means of AHP and Heuristic Evaluation (HE). Wey and Chiu (2013) did a user-centered research to assess today's pedestrian needs for transit oriented environment design by using the House of Quality matrix (HOQ) technique combined with ANP. 
Raharjo et al. (2008) used QFD based on ANP as a customer driven tool to deal with subjectivity issues in early product design phase.

It is seen that the methods are used for different tasks including evaluation, selection and decision making; they are used in conjunction with other methods and tools; they are used at various stages of the design process and not only at the final stage for selection among alternatives; they are used for critical decision making not only regarding designs but also regarding design-related activities and process-related issues. The popularity of the methods to be used in so many different purposes and fields can be seen as a sign of their value and versatility. While some of the studies we encountered in our own search of the literature occasionally use the words product design, they usually referred to the engineering aspects of product development. Therefore the studies seem to focus on solving mostly the technical issues of design, seen by engineers' eyes. In view of these facts, this article presents an effective, versatile and customizable framework for industrial designers to use AHP and ANP for various needs of their industrial design projects.

\section{ANALYTICAL HIERARCHY PROCESS}

Analytic Hierarchy Process (AHP) was first proposed by Myers and Alpert (1968) and further developed for application in Wharton School of Business (Saaty, 1980), establishing a place for itself as a tool of decision making and priority identification (Vaidya and Kumar, 2006; Cheng et al., 2005; Lai et al., 1999; Min, 1994). AHP is basically a measurement theory based on priority values obtained from pairwise comparisons between criteria and alternatives (Y1lmaz, 1999). It can be used for solving decision making problems in systems having complex relations with its subsystems. It works by analyzing and modeling these systems heuristically as simplified hierarchical structures (Özden, 2008). By using a simplified structure, AHP prevents costly, distractive and delay imposing problems frequently encountered in large decision making processes such as lack of focus, lack of involvement and planning mistakes (Koçak, 2003).

In AHP, the relation between the decision processes is unidirectional and the overall process is comprised of three steps (Wind and Saaty, 1980). For the solution of the problem, first a hierarchical structure is formed (An et al., 2007). Then a pairwise comparison matrix which determines the priorities in terms of the relative importance values of the criteria is calculated (Cao et al., 2008; Başak, 2002). Saaty's Eigenvector method is used to calculate the relative importance values (Garcia-Cascales and Lamata, 2009). Then the consistency of the values in the matrix is checked by calculating the consistency ratio (Chou and Hsu, 2008). If the consistency ratio is between acceptable limits, the process continues with assessing the priorities of the alternatives to see which of the alternative has the highest priority, therefore is the most successful (Y1lmaz, 2010).

The process steps in detail are as follows:

\section{Setting Up the Model (Structuring the Hierarchy)}

Structuring a problem as a hierarchical schema means separation of the problem into different layers. This procedure is called the modeling (Peng and Dai, 2009; Chandran et al., 2005). Modeling gives the decision makers the opportunity to effectively see and compare the criteria, sub criteria and alternatives (Lee and Hwang, 2010). The purpose of the overall evaluation 


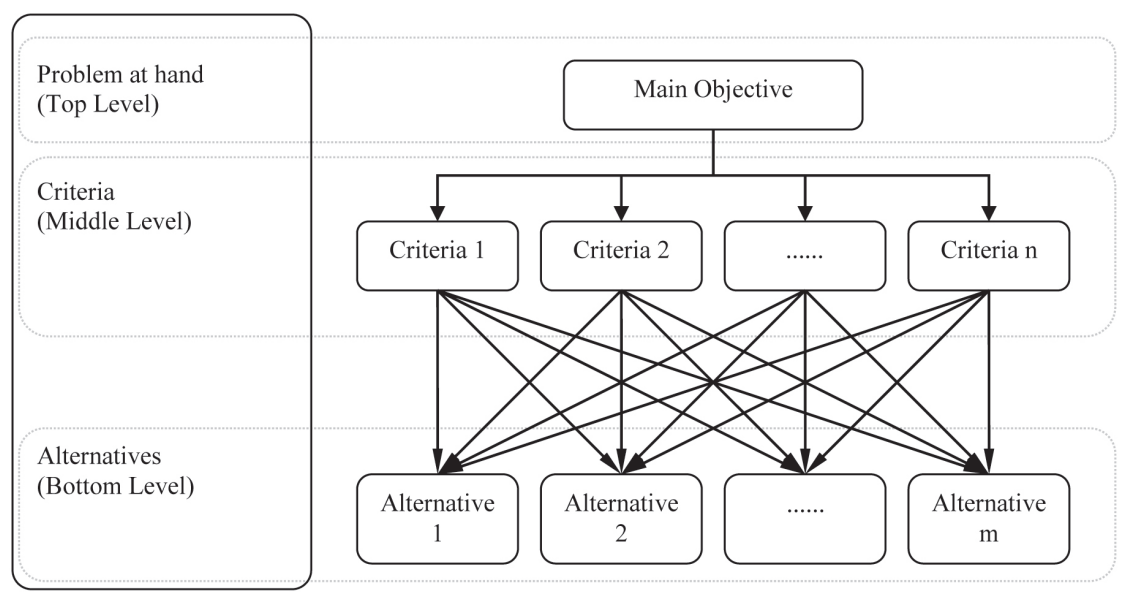

procedure is written at the top of the hierarchy (Pineda-Henson et al., 2008). Below this level, the criteria to be used in the evaluation are listed and at the next level alternatives to be evaluated are listed (Braunschweig and Becker, 2004). The resulting hierarchical structure can be seen in Figure 1 (Wang, Liu and Elhag, 2008).

\section{Forming the Pairwise Comparison Matrices and Designating the Weighted Values}

In the second step of AHP, the pairwise comparison matrices are formed and the relative importance value of each criteria is investigated (Chandran et al., 2005). These values are found by doing pairwise comparisons by the persons taking part in the study (Sharma et al.,2008). Knowledge and experience of the participants are important for the efficiency of the comparisons (Chandran et al., 2005). The pairwise comparison matrix for the criteria is shown in Table 1.

Table 1. The pairwise comparison matrix for the criteria

Table 2. Relative importance 1-9 scale used in AHP and their definitions

\begin{tabular}{|c|c|c|c|c|}
\hline & Criteria 1 & Criteria 2 & Criteria ... & Criteria j \\
\hline Criteria 1 & $\frac{w_{1}}{w_{1}}$ & $\frac{w_{1}}{w_{2}}$ & $\ldots$ & $\frac{w_{1}}{w_{j}}$ \\
\hline Criteria 2 & $\frac{w_{2}}{w_{1}}$ & $\frac{w_{2}}{w_{2}}$ & $\ldots$ & $\frac{w_{2}}{w_{j}}$ \\
\hline Criteria ... & $\ldots$ & $\ldots$ & $\ldots$ & $\ldots$ \\
\hline Criteria i & $\frac{w_{i}}{w_{1}}$ & $\frac{w_{i}}{w_{2}}$ & $\ldots$ & $\frac{w_{i}}{w_{j}}$ \\
\hline w: weighted value / relative importance value
\end{tabular}

\begin{tabular}{|c|l|l|}
\hline $\begin{array}{c}\text { Relative } \\
\text { Importance } \\
\text { Value }\end{array}$ & \multicolumn{1}{|c|}{ Meaning } & \multicolumn{1}{c|}{ Explanation } \\
\hline 1 & Equal value & Two requirements are of equal value \\
\hline 3 & Slightly more value & Experience slightly favors one requirement over the other \\
\hline 5 & Essential or strong value & Experience strongly favors one requirement over the other \\
\hline 7 & Very strong value & A requirement is strongly favored and its dominance is demonstrated in practice \\
\hline 9 & Extreme value & The evidence favoring one over the other is on the highest possible order of affirmation \\
\hline $2,4,6,8$ & Intermediate values & These values should only be used when a compromise is needed. \\
\hline
\end{tabular}




\begin{tabular}{|c|c|}
\hline Formula 1 & $w_{i}=\frac{1}{n} \sum_{j=1}^{n} \frac{a_{i j}}{\sum_{j=1}^{n} a_{i j}}$ \\
\hline Formula 2 & $C R=\frac{C I}{R I}$ \\
\hline Formula 3 & $C I=\frac{\lambda_{\max }-\mathrm{n}}{n-1}$ \\
\hline Formula 4 & $\lambda_{\max }=\frac{1}{\mathrm{n}} \sum_{i=1}^{n} \frac{(A w)_{i}}{w_{i}}$ \\
\hline
\end{tabular}

The next step is to calculate the importance of the criteria relative to each other. The decision maker uses Saaty's 1-9 scale shown in Table 2 to give importance values to criteria pairs by comparing all of them two at a time (Saaty 1986).

Then the overall relative importance sequence of all the criteria is calculated. The preferred method for this calculation is Saaty's Eigenvector method (Hurley, 2001).

\section{Calculation of the Relative Importance of the Criteria-Sub Criteria and the Consistency Ratio}

The Eigenvector is calculated by using Formula 1 given in Table 3 (Ramadhan et al. 1999). The next step is to calculate the consistency ratio (CR) of the comparison matrix (Hafeez et al. 2007). The purpose of this is to determine whether the participant gave consistent information while comparing the criteria. If the CR exceeds 0.10 , this means the matrix data is inconsistent and the comparisons should be reviewed or repeated (Donegan et al., 1992; Stain and Mizzi, 2007). Therefore the consistency of the matrix is inversely proportional to the $C R$ value and the most consistent matrix is achieved with a CR of zero (Jian-Zhong et al., 2008). Saaty and Özdemir (2003) prefer to use Formula 2 to calculate the consistency of the comparison matrix (Zhou and Shi, 2009; Saaty and Özdemir, 2003). Consistency Index (CI) in Formula 2 can be calculated with Formula 3 (Zhou and Shi, 2009). The $\lambda_{\max }$ in Formula 3, which is the maximum Eigen value, is calculated with Formula 4 (Peng and Dai, 2009).

By adding the values obtained by multiplying the relative priorities with the columns of the comparison matrix, the weighted total vector is formed. The arithmetic average of the value obtained by dividing elements of the weighted total vector into the corresponding relative priority value gives $\lambda_{\max }$ value (Güngör and İşler, 2005). The values of RI according to matrix size are given in Table 4 (Karagiannidis et al., 2010; Wang et al., 2008).

For the final solution to the problem, a hybrid vector of priorities that will be used to rank the alternatives is generated by calculating the weighted average of the vectors for the priorities of all the variables. The final priority values of the alternatives obtained by using these averages are called decision points and they are used to form the hybrid vector on

Table 4. Change of random index values according to matrix size which the decision makers can see and easily compare the performances

\begin{tabular}{|c|c|c|c|c|c|c|c|c|c|c|c|c|c|c|c|}
\hline $\mathbf{n}$ & 1 & 2 & 3 & 4 & 5 & 6 & 7 & 8 & 9 & 10 & 11 & 12 & 13 & 14 & 15 \\
\hline RI & 0 & 0 & 0.58 & 0.90 & 1.12 & 1.24 & 1.32 & 1.41 & 1.45 & 1.49 & 1.51 & 1.48 & 1.56 & 1.57 & 1.59 \\
\hline
\end{tabular}


of the alternatives, and therefore can select the option with the highest performance score (Zahedi, 1986; Kuruüzüm and Atsan, 2001).

\section{ANALYTIC NETWORK PROCESS}

Analytic Network Process (ANP) is a method which has been developed and proposed by Saaty for supporting multi criteria decision making processes and its importance and use have improved considerably in the recent years (Dağdeviren and Yüksel, 2007; Saaty and Shih, 2009). ANP method holistically considers the relations between units and unit groups, interdependencies within those groups and the feedbacks between criteria (Dağdeviren and Yüksel, 2007; Saaty and Shih, 2009). This enables the method to solve decision making problems more efficiently and realistically. As shown in Figure 2, ANP method has a web like structure, which precisely models the problems, internal relations between their main and side components and the directions of the relations. Therefore ANP can consider the direct or indirect interaction and feedback between parts of the problem, which are not methodically investigated in AHP.

While AHP treats the decision making problem as a unidirectional hierarchical structure to systematically calculate the priorities of the criteria, ANP can model and analyze more complex structures. In AHP, criteria of the same level are assumed to be independent of each other and the effects between them are ignored. But in real life problems, criteria that affect a decision usually also affect other criteria. As shown in Figure 2, ANP appraises these interior relations (Dağdeviren et al., 2006) by modeling the decision problem like a web in which exterior dependencies and feedbacks between criteria groups, as well as interior dependencies within the groups are considered. This enables more complex problems to be modeled that would be hard to model with a hierarchical structure and provides the evaluations or decisions to be more precise and effective (Karsak et al., 2002).

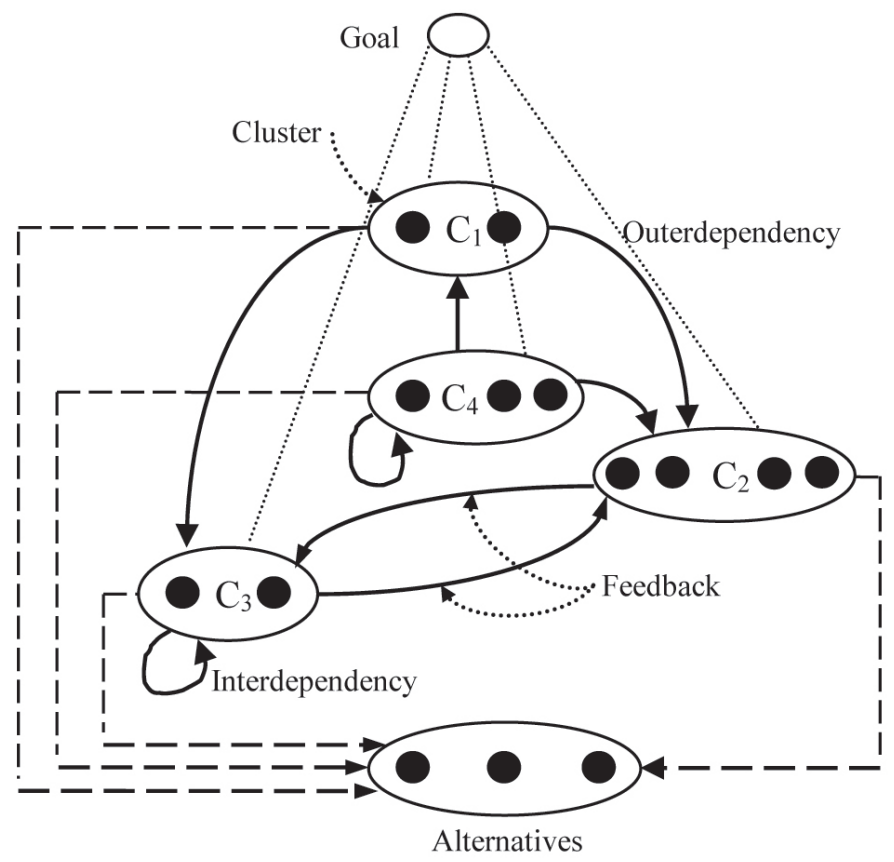


Priority vectors are calculated in both methods by using dual comparison matrices but while the AHP procedure works in a sequential way, ANP follows a more complex route. In ANP, the relations between the criteria clusters (external dependency) or mutual effects between criteria belonging to the same cluster (internal dependency) are calculated as vectors and added to a comparison matrix as a column. As a criteria cluster does not necessarily affect another criteria cluster or a single criteria all the time, values belonging to these kinds of neutral criteria in the vector are taken as zero (Büyükyazıcı and Sucu, 2003).

The process steps in detail are as follows (Bayazit, 2006).

\section{Determining the Main Purpose and Forming the Model}

The criteria and alternatives are designated and listed. They are classified into separate clusters according to their interior relations. Then the ANP web structure is formed by investigating and considering interactions and dependencies between clusters.

\section{Forming of the Dual Comparison Matrices and Calculating the Eigen Vector}

Pairwise comparison data obtained from interviews with participants are entered into matrices by using the same 1-9 scale also used in the AHP method.

If the consistency ratio calculated from the pairwise comparison data is below 0.1 , the evaluations performed by the participants are accepted to be sufficiently consistent and therefore correct. The value in the matrix belonging to a criterion which is found to have no relation with any other criterion is taken as zero. This enables the Eigen vector to be calculated without problem at all times. An un-weighted super matrix is formed by placing the Eigen vectors into the columns of the formed matrix.

$$
A=\left(a_{i j}\right)_{n x n}=\left[\begin{array}{cccc}
a_{11} & a_{12} & \ldots & a_{1 n} \\
a_{21} & a_{22} & \ldots & a_{2 n} \\
\cdot & \cdot & \cdot & \cdot \\
a_{n 1} & a_{n 2} & \ldots & a_{n n}
\end{array}\right]
$$

\section{Calculation of the Weighted Super Matrix}

A new matrix is formed by multiplying the values in the un-weighted super matrix with the weights of the cluster they belong to and this new matrix is called the weighted super matrix. If the sum of the columns of the weighted super matrix is not equal to 1 , a normalization operation needs to be done to equalize this sum to 1 . To equalize the priorities at one point, the matrix is raised to powers to capture all transitivity of an order that is equal to that power. The new matrix formed is called the limit super matrix.

\section{Organizing the Alternatives and Reaching the Results}

The final priorities of the criteria and the alternatives are calculated by normalizing each cluster. Then the alternatives are organized in a vector from high to low according to their priority values. 


\section{USING AHP AND ANP FOR THE EVALUATION OF HAND CART DESIGN CONCEPTS}

In this section, a case study is presented to demonstrate the applicability and validity of AHP and ANP methods.

\section{The Application}

A number of hand cart design concepts are evaluated by using the two methods and one of them is selected for further design development and production. For this, the criteria which may affect buyers' hand cart selection preferences are listed and the priorities of these criteria among each other are assessed. Then these criteria are used for evaluating the hand cart design concepts by using the AHP and ANP methods and thus the final concept is selected. Results obtained from the two methods are compared and discussed.

\section{The Method}

After investigating the utilization of AHP and ANP in the literature, our adaptation of the two methods into industrial design is planned. It is seen that the AHP and ANP methods are known to work with both objective as well as subjective input data (Kuruüzüm and Atsan, 2001) and according to the literature, there is no strict number for the amount of samples required. Therefore 15 people attending the university's design research center were randomly selected to take part in the study, consisting of a professor and eight designers to represent design experts and six design students to represent users (referred to as participants from here on). One on one interviews were conducted with the participants to give scores for the priorities of the criteria and the alternatives. To create a common decision for the whole sample group, the geometric mean of the input data was calculated for both methods (Saaty, 2004; Saaty, 2008). Storage and processing of the data gathered from the interviews were done on separate PC software dedicated to each method and the findings were obtained separately.

\section{The Common Tasks of the Two Methods}

As ANP is derived from AHP, initial parts of the processes are similar. Both methods operate around a main objective and use a set of criteria and sub criteria to perform the evaluations. So the main objective was defined first as "selection of the best hand cart design concept". This implies selecting the design which will have the greatest chance of being successful in market, creating the highest profit. Then the criteria and sub criteria were selected and gathered from literature articles that used various methods for similar evaluations (Hambali et al., 2008; Hambali et al., 2009; Ayağ, 2005; Hsiao et al., 2002; Xiao et al., 2007). As shown in Table 5, the selected main criteria are: performance, safety, cost, human factors, and maintenance and the self-explanatory sub criteria are: ease of transfer, ease of operation, ease of storage, lightness, frame strength, stability, sharp edge elimination, material cost, manufacturing process cost, ease of holding, user friendly handles and locks, ease of repair, and ease of dismantling.

Then, as the design alternatives to be investigated, seven hand cart design concepts shown in Figure 3 were selected from among a number of designs that were investigated in an earlier study (Bayrakçı, 2004).

The brief specifications of the seven selected design concepts are as follows. 


\begin{tabular}{|l|l|}
\hline Performance & $\begin{array}{l}\text { Properties that can affect the general working and usability of } \\
\text { the hand cart, such as ease of carrying, ease of operation, fold- } \\
\text { ing for storage, weight of the product, having a sturdy frame } \\
\text { to carry heavier loads. }\end{array}$ \\
\hline Safety & $\begin{array}{l}\text { Properties that can protect the user from harm or the product } \\
\text { itself from damage such as, being able to stand alone or move } \\
\text { in a stable straight line without rolling or falling, not having } \\
\text { any sharp edges that might harm the user or the goods being } \\
\text { carried. }\end{array}$ \\
\hline Cost & $\begin{array}{l}\text { Purchase price of the product is affected from the cost of } \\
\text { materials used in manufacture and the cost of manufactur- } \\
\text { ing itself and this is always an important factor affecting any } \\
\text { purchase. }\end{array}$ \\
\hline Human Factors & $\begin{array}{l}\text { Properties related to user-product physical interface, product } \\
\text { dimensions compatible with anthropometric measures, ease } \\
\text { of holding, pulling, opening-closing and folding-unfolding } \\
\text { the product for daily use and storage. }\end{array}$ \\
\hline Maintenance & $\begin{array}{l}\text { The periodical servicing that the product may need, the inter- } \\
\text { val of this necessary servicing, the possibility of the servicing } \\
\text { to be performed by the user, whether the interior details of the } \\
\text { product are easily seen and reachable to enable problem find- } \\
\text { ing and the possibility of the product to be dismantled easily } \\
\text { to reach the faulty point. }\end{array}$ \\
\hline
\end{tabular}

HC-1 is a soft bag design with a flexible lid, hard bottom and a rectangular frame, which can be pulled or pushed by a handle or carried by two shoulder straps as a backpack.

HC-2 is an open top folding bag supported by four solid fence sides and a diagonal frame.

HC-3 is a basket type bag with semi rigid sides and lid, supported by a strong frame.

HC-4 is a folding bag with minimal frame and small wheels, and has the smallest dimensions among the seven when folded.

HC-5 is a bag with expandable body, rigid top and bottom with telescopic side frames and a carrying handle.

HC-6 is a handcart made from a rectangular strong frame carried by two big wheels and it houses two detachable smaller bags.
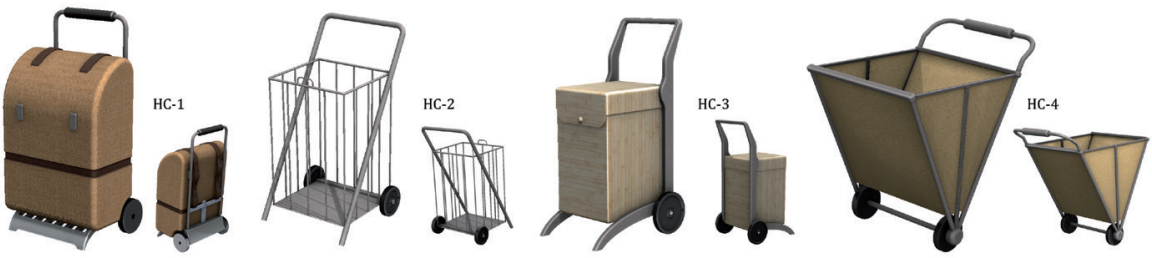

Figure 3. The seven hand cart design concepts, one of which to be selected for further design development (front and rear perspective
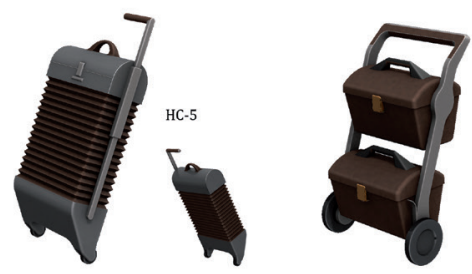

HC-6
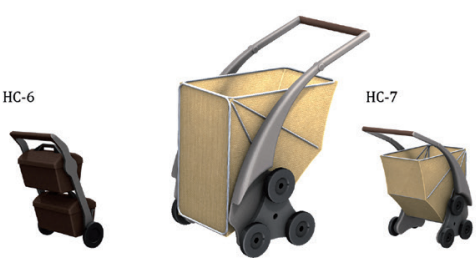
HC-7 is a flexible folding bag with supporting rigid frames and multiple wheels for easy operation on steps.

For the interviews, a private room was prepared with a computer, a table and two seats that the participant and the interviewer used while the participant was asked to do the required comparisons. The interviews usually took between 45 and 60 minutes each. The pictures of the alternatives were shown two by two to the participants on a 23 inch LCD screen for the comparisons. First HC-1 and HC-2 were shown and compared, then HC-1 and HC-3, followed by HC-1 and HC-4. After completing the comparison of the $\mathrm{HC}-1$ and $\mathrm{HC}-7$ pair, the routine restarted for the HC-2 and HC-3 pair, continuing with $\mathrm{HC}-2$ and $\mathrm{HC}-4$ up to HC-2 and HC-7, and then for the pairs of HC-3 and HC-4, HC-3 and $\mathrm{HC}-5$, and so on. The comparison process always followed this same order for every participant. Each comparison was orally given to the participant as a question like "Please state the importance values for performance and safety, which one do you think is more important for a hand cart?", "Which one of the two displayed design concepts is safer to use? Please comparatively score the displayed design concepts according to safety". This oral and visual approach enabled the surveys to be performed easily like an informal conversation. The number of necessary comparisons between $n$ items can be calculated by the combination formula $n *(n-1) / 2$ (Godwin, 2000). As there were seven alternatives to be compared, $7 *(7-1) / 2=21$ comparisons were necessary for each criterion. And as there were 13 sub criteria under the main criteria, $21 * 13=283$ comparisons were performed for the alternatives. Additional $5 *(5-1) / 2=10$ comparisons were performed among the main criteria and 14 comparisons among the sub criteria, increasing the total comparison number to 307. The participants were asked to do the scoring by using Saaty's 1-9 scale. Received scores were simultaneously entered into the Expert Choice (for AHP) and the ANP Solver (for ANP) programs by the interviewer for later calculations, saving additional time.

\section{The Application of AHP}

After the completion of the common tasks, the study proceeded with method specific tasks. To begin with, a hierarchical structure was formed as shown in Figure 4, in which the main objective of the study was written at the top, the criteria-sub criteria were listed at mid level and the alternatives to be evaluated were listed at the bottom. The criteria and sub criteria were used as the input for the Expert Choice PC program (Version 11). Empty pairwise comparison matrices produced by the program were filled with data gathered from the interviews in which the participants comparatively evaluated the criteria and the alternatives in pairs by using the 1-9 scale shown in Table 2.

When the interviews were complete, the AHP calculations were done by the PC program. As the number of participants was more than one, the program calculated geometric mean average of the values obtained from the interviews for use at the computations. The averages found for the criteria are listed in Table 6 . The inconsistency ratio belonging to the criteria comparisons matrix was found to be 0.00 , while a value below 0.1 implies a consistent result.

According to the findings presented in Figure 5, the participants found the most important criteria for the hand cart design concept to be human 
Figure 4. The hierarchical model of the process

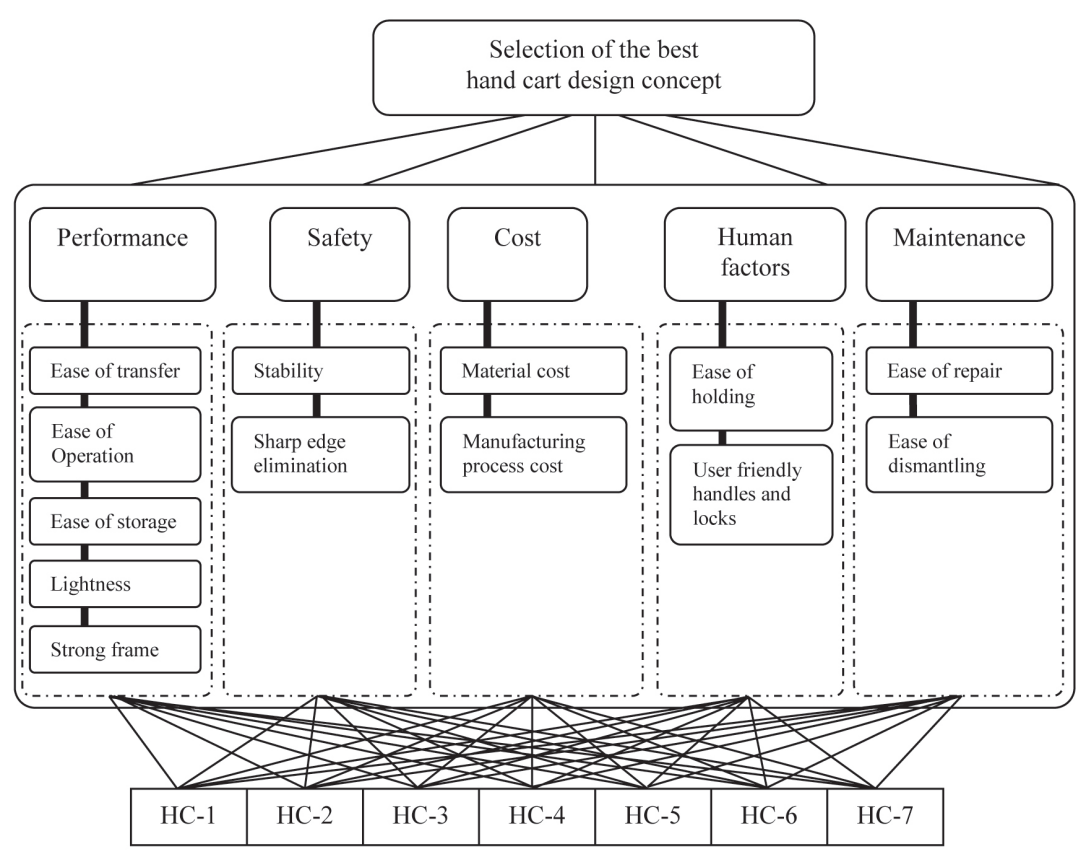

\begin{tabular}{|l|c|c|c|c|c|}
\hline & Performance & Safety & Cost & Human factors & Maintenance \\
\hline Performance & & 1.16591 & 1.08447 & 1.14471 & 1.55185 \\
\hline Safety & & & 1.03996 & 1.35566 & 1.52767 \\
\hline Cost & & & & 1.09682 & 1.22106 \\
\hline Human factors & & & & & 2.13354 \\
\hline Maintenance & & & & & \\
\hline Inconsistency Ratio:0,00 & & & & & \\
\hline
\end{tabular}

Table 6. Pairwise comparison matrix for the main criteria

Figure 5. Score order of the criteria with respect to the objective

Human factors

Performance

Cost

Safety

Maintenance

Inconsistency $=0,00463$

with 0 missing judgments. factors and this was followed by performance, cost, safety and finally maintenance.

After the main criteria, pairwise comparisons were performed for the sub criteria. The inconsistency ratio for the comparisons was calculated as less than 0.1. As can be seen in Figure 6, the most important sub criterion of the criterion of performance was found to be ease of operation, which was followed by ease of transfer, frame strength, ease of storage and lightness. The most important sub criterion of the criterion of safety came up as stability, while the most important sub criterion of cost was material cost, the most important sub criterion for human factors was ease of holding, and the most important sub criterion for maintenance was ease of dismantling.

Overall Inconsistency $=, 01$

Figure 7. Decision points of the alternatives

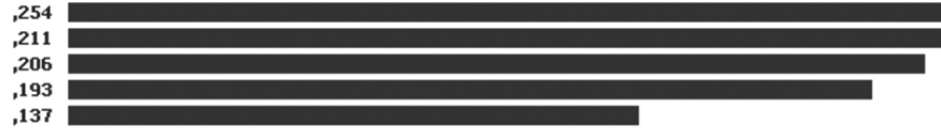


Figure 6. Hierarchical schema of importance values for the criteria and sub criteria
Table 7. Dependency determination matrix for the main criteria

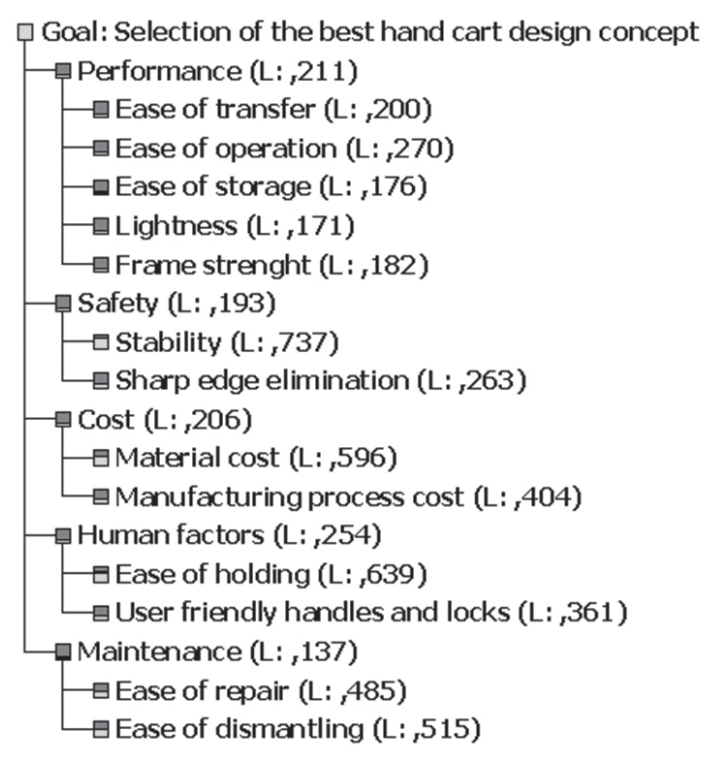

The resulting decision points (weighted importance values) for the seven hand cart alternatives are listed in Figure 7. It is seen that HC-4 has the highest overall performance score $(16.1 \%)$ and HC-1 has the second highest $(16 \%)$, while HC-6 has the lowest $(11.9 \%)$. Therefore after considering all criteria and sub criteria, HC-4 was found to be the most promising design concept alternative for further design development. This was the hand cart design concept with folding bag on minimal frame and small wheels.

\section{The Application of ANP}

The same set of criteria and sub criteria were also used for ANP. As the main difference of the ANP method is to consider the internal and external dependencies between criteria and sub criteria, these dependencies were also determined by consulting the participant professor in a separate interview session. In other sessions, he also participated in the study for comparing the $\mathrm{HC}$ alternatives as a design expert. Obtained dependency data is saved on dependency determination matrices provided by the ANP solver program shown in Table 7 and Table 8. Whenever there is any dependency between left column and first line elements, "true" is displayed at the corresponding cell and "false" otherwise. If the column and the line element is the same and the cell says true, this means that there is interdependency between its sub criteria. The dependency schematic generated by the PC program by using the dependency matrices is shown in Figure 8; in this figure the straight arrows show the relations in between criteria groups and the round arrows show interdependencies.

\begin{tabular}{|l|c|c|c|c|c|c|}
\hline \multicolumn{1}{|c|}{ Clusters } & Performance & Safety & Cost & Human factors & Maintenance & Alternatives \\
\hline Performance & True & True & True & True & True & True \\
\hline Safety & True & False & True & True & True & True \\
\hline Cost & True & True & False & True & True & True \\
\hline Human factors & True & True & True & True & True & True \\
\hline Maintenance & True & True & True & True & True & False \\
\hline Alternatives & True & True & True & True & True & \\
\hline
\end{tabular}




\begin{tabular}{|c|c|c|c|c|c|c|c|c|c|c|c|c|c|c|c|c|c|c|c|c|}
\hline $\begin{array}{l}Z \\
O \\
0 \\
D \\
\infty \\
\infty\end{array}$ & 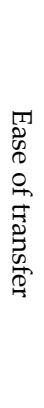 & $\begin{array}{l}\text { T1 } \\
2 \\
0 \\
0 \\
0 \\
0 \\
0 \\
0 \\
\mathbb{0} \\
0 \\
0 \\
0 \\
0 \\
0\end{array}$ & $\begin{array}{l}1 \\
\text { T1 } \\
0 \\
0 \\
0 \\
0 \\
0 \\
0 \\
0 \\
0 \\
0 \\
0\end{array}$ & 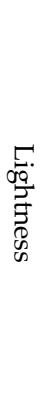 & $\begin{array}{l}T \\
\mathbb{3} \\
0 \\
0 \\
0 \\
0 \\
0 \\
0 \\
00 \\
0 \\
0\end{array}$ & 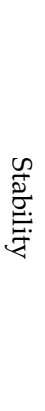 & 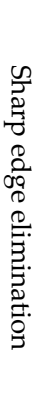 & 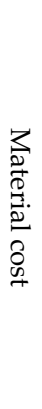 & 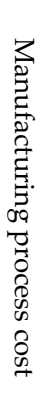 & 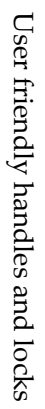 & 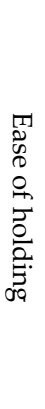 & 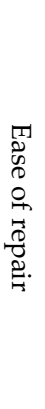 & 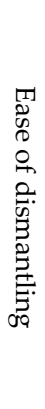 & $\stackrel{T}{\stackrel{T}{S}}$ & 氺 & 罩 & $\stackrel{\mathbb{T}}{\mathfrak{R}}$ & $\begin{array}{l}\mathbb{T} \\
\stackrel{3}{G}\end{array}$ & $\stackrel{T}{\mathfrak{T}}$ & $\stackrel{T}{1}$ \\
\hline Ease of transfer & $\mathrm{F}$ & $\mathrm{T}$ & $\mathrm{F}$ & $\mathrm{T}$ & F & $\mathrm{T}$ & $\mathrm{T}$ & $\mathrm{F}$ & F & $\mathrm{T}$ & $\mathrm{T}$ & $\mathrm{F}$ & $\mathrm{T}$ & $\mathrm{T}$ & $\mathrm{T}$ & $\mathrm{T}$ & $\mathrm{T}$ & $\mathrm{T}$ & $\mathrm{T}$ & $\mathrm{T}$ \\
\hline Ease of operation & $\mathrm{T}$ & F & $\mathrm{F}$ & $\mathrm{T}$ & $\mathrm{T}$ & $\mathrm{T}$ & $\mathrm{T}$ & F & $\mathrm{F}$ & $\mathrm{T}$ & $\mathrm{T}$ & $\mathrm{T}$ & F & $\mathrm{T}$ & $\mathrm{T}$ & $\mathrm{T}$ & $\mathrm{T}$ & $\mathrm{T}$ & $\mathrm{T}$ & $\mathrm{T}$ \\
\hline Ease of storage & $\mathrm{F}$ & F & $\mathrm{F}$ & $\mathrm{T}$ & F & $\mathrm{T}$ & $\mathrm{T}$ & $\mathrm{F}$ & F & F & $\mathrm{T}$ & $\mathrm{F}$ & $\mathrm{T}$ & $\mathrm{T}$ & $\mathrm{T}$ & $\mathrm{T}$ & $\mathrm{T}$ & $\mathrm{T}$ & $\mathrm{T}$ & $\mathrm{T}$ \\
\hline Lightness & $\mathrm{T}$ & $\mathrm{T}$ & $\mathrm{T}$ & $\mathrm{F}$ & F & $\mathrm{F}$ & F & $\mathrm{T}$ & $\mathrm{T}$ & $\mathrm{T}$ & $\mathrm{F}$ & $\mathrm{T}$ & $\mathrm{T}$ & $\mathrm{T}$ & $\mathrm{T}$ & $\mathrm{T}$ & $\mathrm{T}$ & $\mathrm{T}$ & $\mathrm{T}$ & $\mathrm{T}$ \\
\hline Frame strength & $\mathrm{F}$ & $\mathrm{T}$ & $\mathrm{F}$ & $\mathrm{F}$ & $\mathrm{F}$ & $\mathrm{F}$ & F & $\mathrm{T}$ & $\mathrm{T}$ & $\mathrm{F}$ & F & $\mathrm{T}$ & $\mathrm{F}$ & $\mathrm{T}$ & $\mathrm{T}$ & $\mathrm{T}$ & $\mathrm{T}$ & $\mathrm{T}$ & $\mathrm{T}$ & $\mathrm{T}$ \\
\hline Stability & $\mathrm{T}$ & $\mathrm{T}$ & $\mathrm{T}$ & $\mathrm{F}$ & F & F & F & F & F & $\mathrm{T}$ & $\mathrm{T}$ & $\mathrm{F}$ & $\mathrm{T}$ & $\mathrm{T}$ & $\mathrm{T}$ & $\mathrm{T}$ & $\mathrm{T}$ & $\mathrm{T}$ & $\mathrm{T}$ & $\mathrm{T}$ \\
\hline Sharp edge elimination & $\mathrm{T}$ & $\mathrm{T}$ & $\mathrm{T}$ & $\mathrm{F}$ & F & F & $\mathrm{F}$ & $\mathrm{F}$ & $\mathrm{T}$ & $\mathrm{T}$ & $\mathrm{T}$ & $\mathrm{T}$ & $\mathrm{T}$ & $\mathrm{T}$ & $\mathrm{T}$ & $\mathrm{T}$ & $\mathrm{T}$ & $\mathrm{T}$ & $\mathrm{T}$ & $\mathrm{T}$ \\
\hline Material cost & $\mathrm{F}$ & $\mathrm{F}$ & F & $\mathrm{T}$ & $\mathrm{T}$ & $\mathrm{F}$ & $\mathrm{F}$ & $\mathrm{F}$ & $\mathrm{F}$ & $\mathrm{T}$ & $\mathrm{F}$ & $\mathrm{T}$ & $\mathrm{F}$ & $\mathrm{T}$ & $\mathrm{T}$ & $\mathrm{T}$ & $\mathrm{T}$ & $\mathrm{T}$ & $\mathrm{T}$ & $\mathrm{T}$ \\
\hline Manufacturing process cost & $\mathrm{F}$ & F & $\mathrm{F}$ & $\mathrm{T}$ & $\mathrm{T}$ & $\mathrm{F}$ & $\mathrm{T}$ & $\mathrm{F}$ & F & $\mathrm{T}$ & $\mathrm{F}$ & $\mathrm{F}$ & $\mathrm{F}$ & $\mathrm{T}$ & $\mathrm{T}$ & $\mathrm{T}$ & $\mathrm{T}$ & $\mathrm{T}$ & $\mathrm{T}$ & $\mathrm{T}$ \\
\hline User friendly handles and locks & $\mathrm{T}$ & $\mathrm{T}$ & $\mathrm{T}$ & $\mathrm{F}$ & F & $\mathrm{T}$ & $\mathrm{T}$ & $\mathrm{T}$ & $\mathrm{T}$ & F & $\mathrm{T}$ & $\mathrm{F}$ & $\mathrm{T}$ & $\mathrm{T}$ & $\mathrm{T}$ & $\mathrm{T}$ & $\mathrm{T}$ & $\mathrm{T}$ & $\mathrm{T}$ & $\mathrm{T}$ \\
\hline Ease of holding & $\mathrm{T}$ & $\mathrm{T}$ & $\mathrm{T}$ & $\mathrm{F}$ & F & $\mathrm{T}$ & $\mathrm{T}$ & $\mathrm{F}$ & F & $\mathrm{T}$ & $\mathrm{F}$ & F & $\mathrm{T}$ & $\mathrm{T}$ & $\mathrm{T}$ & $\mathrm{T}$ & $\mathrm{T}$ & $\mathrm{T}$ & $\mathrm{T}$ & $\mathrm{T}$ \\
\hline Ease of repair & $\mathrm{F}$ & $\mathrm{T}$ & $\mathrm{F}$ & $\mathrm{T}$ & $\mathrm{T}$ & $\mathrm{F}$ & $\mathrm{T}$ & $\mathrm{T}$ & F & $\mathrm{F}$ & $\mathrm{F}$ & $\mathrm{F}$ & $\mathrm{T}$ & $\mathrm{T}$ & $\mathrm{T}$ & $\mathrm{T}$ & $\mathrm{T}$ & $\mathrm{T}$ & $\mathrm{T}$ & $\mathrm{T}$ \\
\hline Ease of dismantling & $\mathrm{T}$ & F & $\mathrm{T}$ & $\mathrm{T}$ & F & $\mathrm{T}$ & $\mathrm{T}$ & $\mathrm{F}$ & $\mathrm{F}$ & $\mathrm{T}$ & $\mathrm{T}$ & $\mathrm{T}$ & $\mathrm{F}$ & $\mathrm{T}$ & $\mathrm{T}$ & $\mathrm{T}$ & $\mathrm{T}$ & $\mathrm{T}$ & $\mathrm{T}$ & $\mathrm{T}$ \\
\hline $\mathrm{HC}-1$ & $\mathrm{~T}$ & $\mathrm{~T}$ & $\mathrm{~T}$ & $\mathrm{~T}$ & $\mathrm{~T}$ & $\mathrm{~T}$ & $\mathrm{~T}$ & $\mathrm{~T}$ & $\mathrm{~T}$ & $\mathrm{~T}$ & $\mathrm{~T}$ & $\mathrm{~T}$ & $\mathrm{~T}$ & $\mathrm{~F}$ & $\mathrm{~F}$ & $\mathrm{~F}$ & $\mathrm{~F}$ & F & $\mathrm{F}$ & $\mathrm{F}$ \\
\hline $\mathrm{HC}-2$ & $\mathrm{~T}$ & $\mathrm{~T}$ & $\mathrm{~T}$ & $\mathrm{~T}$ & $\mathrm{~T}$ & $\mathrm{~T}$ & $\mathrm{~T}$ & $\mathrm{~T}$ & $\mathrm{~T}$ & $\mathrm{~T}$ & $\mathrm{~T}$ & $\mathrm{~T}$ & $\mathrm{~T}$ & $\mathrm{~F}$ & $\mathrm{~F}$ & $\mathrm{~F}$ & F & $\mathrm{F}$ & $\mathrm{F}$ & $\mathrm{F}$ \\
\hline HC-3 & $\mathrm{T}$ & $\mathrm{T}$ & $\mathrm{T}$ & $\mathrm{T}$ & $\mathrm{T}$ & $\mathrm{T}$ & $\mathrm{T}$ & $\mathrm{T}$ & $\mathrm{T}$ & $\mathrm{T}$ & $\mathrm{T}$ & $\mathrm{T}$ & $\mathrm{T}$ & F & $\mathrm{F}$ & F & F & F & F & $\mathrm{F}$ \\
\hline $\mathrm{HC}-4$ & $\mathrm{~T}$ & $\mathrm{~T}$ & $\mathrm{~T}$ & $\mathrm{~T}$ & $\mathrm{~T}$ & $\mathrm{~T}$ & $\mathrm{~T}$ & $\mathrm{~T}$ & $\mathrm{~T}$ & $\mathrm{~T}$ & $\mathrm{~T}$ & $\mathrm{~T}$ & $\mathrm{~T}$ & F & F & F & F & F & F & $\mathrm{F}$ \\
\hline HC-5 & $\mathrm{T}$ & $\mathrm{T}$ & $\mathrm{T}$ & $\mathrm{T}$ & $\mathrm{T}$ & $\mathrm{T}$ & $\mathrm{T}$ & $\mathrm{T}$ & $\mathrm{T}$ & $\mathrm{T}$ & $\mathrm{T}$ & $\mathrm{T}$ & $\mathrm{T}$ & F & F & F & F & F & F & $\mathrm{F}$ \\
\hline HC-6 & $\mathrm{T}$ & $\mathrm{T}$ & $\mathrm{T}$ & $\mathrm{T}$ & $\mathrm{T}$ & $\mathrm{T}$ & $\mathrm{T}$ & $\mathrm{T}$ & $\mathrm{T}$ & $\mathrm{T}$ & $\mathrm{T}$ & $\mathrm{T}$ & $\mathrm{T}$ & F & $\mathrm{F}$ & $\mathrm{F}$ & F & F & F & $\mathrm{F}$ \\
\hline HC-7 & $\mathrm{T}$ & $\mathrm{T}$ & $\mathrm{T}$ & $\mathrm{T}$ & $\mathrm{T}$ & $\mathrm{T}$ & $\mathrm{T}$ & $\mathrm{T}$ & $\mathrm{T}$ & $\mathrm{T}$ & $\mathrm{T}$ & $\mathrm{T}$ & $\mathrm{T}$ & F & F & $\mathrm{F}$ & F & F & F & $\mathrm{F}$ \\
\hline
\end{tabular}

Table 8. Dependency determination matrix for the sub criteria
After determining the dependencies, the 15 participants were asked to carry out pairwise comparisons using the 1-9 scale which was also used for the AHP method. Then the geometric average of this data was calculated and entered to the ANP solver program (ANP Solver, 2016) to obtain the final results. As the inconsistency rates for all of the matrices were calculated as lower than 0.1 by the program, the evaluations were accepted as valid. Table 9 shows the un-weighted super matrix, with the calculated Eigenvectors.

The weighted matrix was next formed by multiplying the values in this matrix to the weight of the related set. If the sum of the values at each column of the weighted super matrix does not add up to 1, normalization should be performed. Table 10 shows the weighted super matrix. As the 


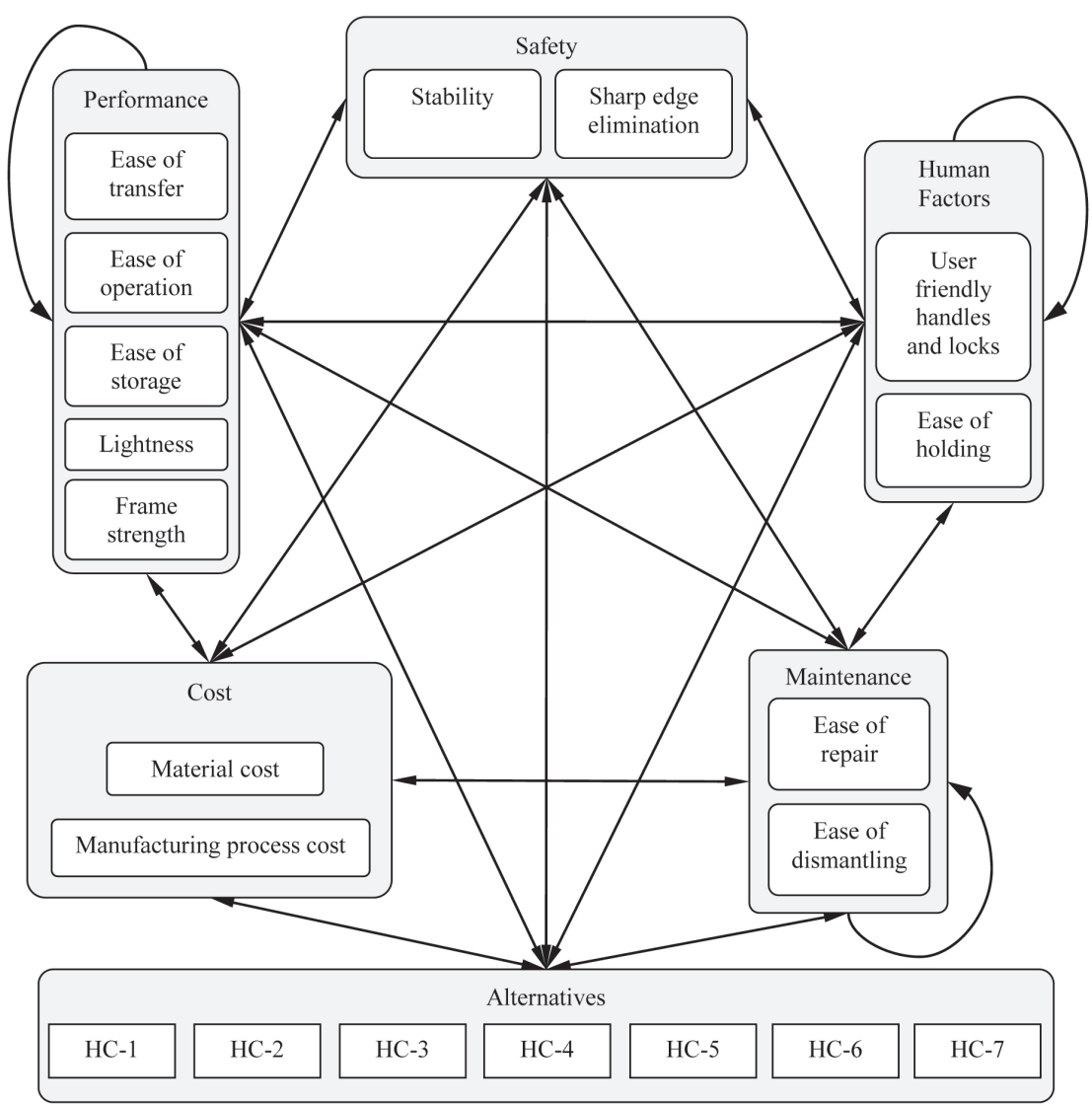

sum of the column values all added up to 1, it was not necessary to perform normalization.

To equalize the importance weights at a point, the $(2 n+1)^{\text {th }}$ power of the super matrix is calculated. The " $n$ " value is a randomly selected large number. The resulting matrix shown in Table $\mathbf{1 1}$ is called the limit super matrix and it lists the weights of the importance values belonging to criteria, sub criteria and design concept alternatives that were compared in the process. The design concept with the highest importance value is the strongest candidate to be selected for further development, and the criteria with the highest importance value is the one having the most effect in the decision making process.

Among the hand cart design concept alternatives listed in Table 12, HC-1 $(0,194)$ has the highest overall performance score, HC-4 $(0,145)$ has average and HC-7 $(0,105)$ has the lowest performance score. The most important criterion is safety $(0,41)$, followed by performance $(0,306)$. The most important sub criterion for performance is ease of transfer $(0,271)$, the most important sub criterion for safety is stability $(0,734)$, the most important sub criterion for cost is material cost $(0,603)$, the most important sub criterion for human factors is user friendly handles and locks $(0,637)$, and the most important sub criterion for maintenance is ease of repair $(0,581)$. Overall performance scores of all of the alternatives for both methods are listed in Table 13. 


\begin{tabular}{|c|c|c|c|c|c|c|c|c|c|c|c|c|c|c|c|c|c|c|c|c|}
\hline HC-7 & $\begin{array}{l}\text { bे } \\
\text { స̦ } \\
0\end{array}$ & స్ & $\frac{\infty}{\infty}$ & $\stackrel{0}{0}$ & $\stackrel{H}{5}$ & 总 & 孚 & $\begin{array}{l}\stackrel{m}{0} \\
\stackrel{0}{0}\end{array}$ & $\begin{array}{l}\infty \\
\infty \\
c_{0}\end{array}$ & $\stackrel{0}{0}_{0}^{\infty}$ & $\begin{array}{l}\tilde{\sigma} \\
\tilde{~} \\
\delta\end{array}$ & 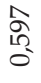 & 畩 & 0 & 0 & 0 & 0 & 0 & 0 & 0 \\
\hline HC-6 & $\begin{array}{l}\text { त् } \\
\text { ¿ }\end{array}$ & त్ & $\stackrel{\infty}{\infty}$ & $\begin{array}{l}\infty \\
0 \\
0 \\
0\end{array}$ & $\frac{1}{2}$ & क्ञ & $\begin{array}{l}\text { Bo } \\
0 \\
0\end{array}$ & $\vec{\Xi}$ & $\begin{array}{l}\infty \\
\substack{0 \\
0 \\
0}\end{array}$ & $\stackrel{\substack{0 \\
0 \\
0}}{0}$ & $\mid \begin{array}{l}\infty \\
\infty \\
0 \\
0\end{array}$ & $\begin{array}{l}\overbrace{0}^{\infty} \\
0 \\
0\end{array}$ & $\begin{array}{l}\text { है } \\
\text { ले }\end{array}$ & 0 & 0 & 0 & 0 & 0 & 0 & 0 \\
\hline HC-5 & 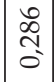 & $\begin{array}{l}\stackrel{1}{2} \\
\text { O } \\
0\end{array}$ & 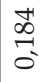 & 今. & 皇 & क्ष & 离 & $\begin{array}{l}\overrightarrow{3} \\
0\end{array}$ & 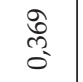 & 兽 & 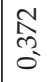 & $\begin{array}{l}0_{0}^{\circ} \\
0 \\
0\end{array}$ & స్ & 0 & 0 & 0 & 0 & 0 & 0 & 0 \\
\hline HC-4 & $\begin{array}{l}\text { ô } \\
\text { o. } \\
0 .\end{array}$ & $\begin{array}{l}\text { N̦ } \\
\text { ָे }\end{array}$ & 疍 & 点 & $\stackrel{9}{0}$ & $\begin{array}{l}\infty \\
\infty \\
1 \\
0 \\
0\end{array}$ & F & $\begin{array}{l}2 \\
2 \\
0 \\
0\end{array}$ & 峉 & $\begin{array}{l}n \\
0 \\
0\end{array}$ & $\mid \begin{array}{l}\infty \\
\infty \\
0 \\
0 \\
0\end{array}$ & . & $\begin{array}{l}\infty \\
\infty \\
0 \\
0\end{array}$ & 0 & 0 & 0 & 0 & 0 & 0 & 0 \\
\hline HC-3 & $\begin{array}{l}\text { ถે } \\
\text { రे }\end{array}$ & $\overrightarrow{\text { สี }}$ & $\begin{array}{l}\infty \\
\stackrel{\infty}{\sigma} \\
=\end{array}$ & $\stackrel{0}{0}$ & $\begin{array}{l}\infty \\
0 \\
\vdots \\
0\end{array}$ & ठే & 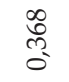 & 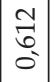 & $\begin{array}{l}\infty \\
\infty \\
\infty \\
0 \\
0\end{array}$ & 丞 & $\begin{array}{l}0 \\
\hat{0} \\
0 \\
0\end{array}$ & $\begin{array}{l}\infty \\
\vdots \\
\text { ch } \\
0\end{array}$ & 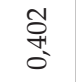 & 0 & 0 & 0 & 0 & 0 & 0 & 0 \\
\hline HC-2 & \begin{tabular}{l}
$\infty$ \\
\multirow{2}{*}{} \\
0 \\
0
\end{tabular} & त् & $\tilde{\delta}$ & \begin{tabular}{|l}
$\hat{A}$ \\
0 \\
0
\end{tabular} & 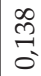 & 齐 & $\begin{array}{l}\text { స̃ } \\
\text { ळ్ }\end{array}$ & 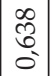 & $\begin{array}{l}\text { No } \\
\text { న. } \\
0\end{array}$ & \begin{tabular}{l}
\multirow{1}{*}{} \\
O̊
\end{tabular} & $\begin{array}{l}0 \\
0 \\
0 \\
0 \\
0\end{array}$ & $\stackrel{8}{0}$ & $\stackrel{n}{\dddot{2}}$ & 0 & 0 & 0 & 0 & 0 & 0 & 0 \\
\hline HC-1 & $\begin{array}{l}\text { त् } \\
\text { ¿ }\end{array}$ & స̃ & $\tilde{\sigma}$ & 点 & $\frac{m}{0}$ & 鸽 & $\begin{array}{l}\text { के } \\
\text { लn } \\
0\end{array}$ & $\left|\begin{array}{c}2 \\
\vec{b} \\
0\end{array}\right|$ & $\begin{array}{l}\vec{\infty} \\
\stackrel{0}{0} \\
0\end{array}$ & त్ & 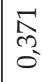 & $\begin{array}{l}\text { है } \\
\text { oे }\end{array}$ & $\begin{array}{l}\vec{\infty} \\
\aleph_{0} \\
0\end{array}$ & 0 & 0 & 0 & 0 & 0 & 0 & 0 \\
\hline $\begin{array}{l}\text { Ease of } \\
\text { dismantling }\end{array}$ & $\begin{array}{l}\text { 芦 } \\
0 \\
0\end{array}$ & 0 & $\begin{array}{l}\text { त̂ } \\
\text { న్ }\end{array}$ & $\mid \begin{array}{c}\hat{m} \\
\tilde{c} \\
\tilde{\sigma}\end{array}$ & 0 & $\begin{array}{l}\text { 壳 } \\
\text { 。 }\end{array}$ & 占 & 0 & 0 & 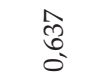 & $\begin{array}{l}0 \\
0 \\
0 \\
0 \\
0\end{array}$ & 0 & 0 & $\begin{array}{l}2 \\
\stackrel{0}{0} \\
0\end{array}$ & 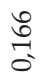 & $\begin{array}{ll}2 \\
0 \\
0\end{array}$ & $\mid \begin{array}{l}\infty \\
\vdots \\
\vdots \\
0\end{array}$ & $\begin{array}{l}2 \\
0 \\
0\end{array}$ & 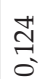 & $\stackrel{8}{\circ}$ \\
\hline Ease of repair & 0 & $\underset{I}{\sharp}$ & 0 & ב̂̃ & \begin{tabular}{l}
+ \\
\multirow{2}{0}{} \\
0
\end{tabular} & 0 & 0 & 0 & 0 & 0 & 0 & 0 & 0 & 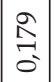 & 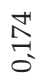 & $\mid \begin{array}{l}\vec{n} \\
- \\
0\end{array}$ & $\left|\begin{array}{l}0 \\
\stackrel{2}{0} \\
0\end{array}\right|$ & $\begin{array}{l}\infty \\
0 \\
0 \\
0\end{array}$ & $\underset{0}{0}$ & 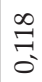 \\
\hline Ease of holding & $\begin{array}{c}\infty \\
\text { ले } \\
\sigma^{\circ}\end{array}$ & 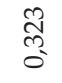 & 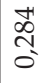 & 0 & 0 & $\begin{array}{l}\tilde{N} \\
\hat{0} \\
0\end{array}$ & 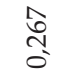 & 0 & 0 & 0 & 0 & 0 & 0 & 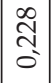 & 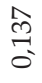 & 胥 & $\mid \begin{array}{c}0 \\
m \\
0\end{array}$ & 足 & స̃ & $\underset{ت}{ت}$ \\
\hline $\begin{array}{l}\text { User friendly } \\
\text { handles and locks }\end{array}$ & $\begin{array}{l}0 \\
2 \\
\tilde{c} \\
0\end{array}$ & $\begin{array}{l}\text { ते } \\
\text { రై }\end{array}$ & 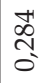 & 0 & 0 & $\begin{array}{l}\hat{\aleph} \\
\hat{\sigma}\end{array}$ & 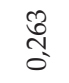 & $\mid \begin{array}{l}0 \\
2 \\
10 \\
0\end{array}$ & 莣 & 0 & 0 & 0 & 0 & $\mid \begin{array}{l}2 \\
5 \\
0 \\
0\end{array}$ & $\begin{array}{l}\frac{8}{0} \\
0\end{array}$ & $\begin{array}{l}1 \\
\stackrel{n}{0} \\
0 \\
0\end{array}$ & $\begin{array}{l}2 \\
0 \\
0\end{array}$ & $\begin{array}{l}\hat{\Xi} \\
\vdots \\
0\end{array}$ & ב̇ & $\begin{array}{l}0 \\
0 \\
0\end{array}$ \\
\hline $\begin{array}{l}\text { Manufacturing } \\
\text { process cost }\end{array}$ & 0 & 0 & 0 & $\mid \begin{array}{l}\infty \\
1+1 \\
10 \\
0\end{array}$ & f̂ & $\circ$ & 0 & 0 & 0 & 0 & $\circ$ & 0 & 0 & $\mid \begin{array}{l}5 \\
5 \\
0\end{array}$ & $\begin{array}{l}\hat{0} \\
\stackrel{0}{0}\end{array}$ & $\begin{array}{l}0 \\
0 \\
0 \\
0\end{array}$ & 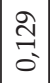 & $\begin{array}{l}\vec{\Xi} \\
\vdots \\
0\end{array}$ & 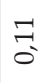 & 吕 \\
\hline Material cost & 0 & 0 & 0 & $\mid \begin{array}{l}\infty \\
\vdots \\
\vdots \\
0\end{array}$ & 点 & o & 0 & 0 & 0 & 0 & 0 & 0 & 0 & $\mid \begin{array}{l}\infty \\
\stackrel{\infty}{-} \\
0\end{array}$ & సิ & $\mid \begin{array}{l}\stackrel{8}{9} \\
-5\end{array}$ & 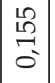 & 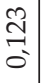 & $\exists$ & 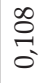 \\
\hline $\begin{array}{l}\text { Sharp edge } \\
\text { elimination }\end{array}$ & $\begin{array}{l}\tilde{\sigma} \\
\text { ले } \\
0\end{array}$ & 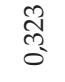 & $\begin{array}{l}\infty \\
\text { तె } \\
0\end{array}$ & 0 & 0 & 0 & 0 & 0 & 0 & 总 & $\begin{array}{l}\infty \\
0 \\
0\end{array}$ & 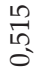 & 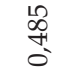 & $\mid$ & $\stackrel{\infty}{0}$ & \begin{tabular}{|l|}
$\vec{n}$ \\
$\overrightarrow{0}$ \\
0
\end{tabular} & $\mid$ & $\begin{array}{l}\text { ô } \\
\text { o. }\end{array}$ & F & F \\
\hline Stability & $\begin{array}{l}0 \\
\infty \\
\infty \\
0\end{array}$ & 岕 & $\frac{2}{0}$ & 0 & $\begin{array}{l}\frac{2}{\infty} \\
0 \\
0\end{array}$ & 壱 & ڤั & 0 & 0 & 菅 & 赑 & 0 & 0 & $\begin{array}{l}\text { ה } \\
0\end{array}$ & ồ & 解 & 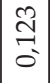 & 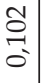 & 然 & 会 \\
\hline Frame strengh & స్ & 芯 & 文 & $\overrightarrow{\overrightarrow{0}}$ & $\begin{array}{l}0 \\
\stackrel{0}{0} \\
0\end{array}$ & o & 0 & 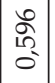 & 落 & 0 & 0 & 0 & 0 & $\mid$ & $\begin{array}{l}\text { L̊ } \\
\text { : }\end{array}$ & 今. & $\begin{array}{l}\text { A } \\
\vdots \\
0\end{array}$ & $\begin{array}{l}\vec{\sigma} \\
0\end{array}$ & 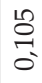 & $\begin{array}{l}2 \\
0 \\
0 \\
0\end{array}$ \\
\hline Lightness & लै & $\stackrel{\widetilde{z}}{0}$ & 交 & $\mid \begin{array}{l}\vec{\infty} \\
\overrightarrow{0}\end{array}$ & $\begin{array}{l}0 \\
\stackrel{5}{0}\end{array}$ & 0 & 0 & 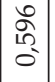 & 莣 & 0 & 0 & . & $\stackrel{\substack{\infty \\
+\infty \\
0}}{0}$ & $\mid \begin{array}{l}5 \\
0 \\
0 \\
0\end{array}$ & $\frac{\infty}{\infty}$ & $\begin{array}{l}\vec{b} \\
\overrightarrow{0}\end{array}$ & 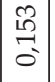 & $\begin{array}{l}\hat{Z} \\
\text { - }\end{array}$ & $\frac{\tilde{d}}{0}$ & 竞 \\
\hline Ease of storage & 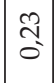 & 芯 & $\begin{array}{l}\infty \\
\stackrel{\infty}{\sigma}\end{array}$ & 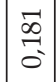 & $\begin{array}{l}8 \\
\stackrel{5}{0}\end{array}$ & 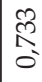 & $\begin{array}{l}\text { ते } \\
\text { స్ }\end{array}$ & 0 & 0 & 0 & 0 & 0 & 0 & $\begin{array}{l}0 \\
\tilde{z} \\
0\end{array} \mid$ & $\begin{array}{l}\text { 至 } \\
\text { 今 }\end{array}$ & $\begin{array}{l}\infty \\
\infty \\
\vdots \\
0\end{array}$ & 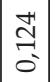 & $\begin{array}{l}\infty \\
0 \\
0 \\
0 \\
0\end{array}$ & $\begin{array}{l}0 \\
\stackrel{1}{0}\end{array}$ & 竞 \\
\hline Ease of operation & 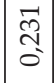 & $\begin{array}{l}\text { त్ } \\
\text { ஸે }\end{array}$ & 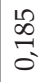 & 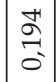 & $\begin{array}{l}\text { 量 } \\
0 \\
0\end{array}$ & 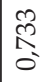 & $\begin{array}{l}\hat{\sigma} \\
\text { ָे }\end{array}$ & 0 & 0 & 芯 & 心్ర & 0 & 0 & $\left|\begin{array}{l}\vec{\infty} \\
\overrightarrow{0} \\
\overrightarrow{0}\end{array}\right|$ & $\begin{array}{l}\stackrel{8}{\circ} \\
\stackrel{\circ}{\circ}\end{array}$ & 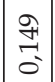 & 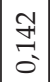 & $\begin{array}{l}\text { సे } \\
\text { ¿े }\end{array}$ & $\begin{array}{l}\vec{I} \\
0\end{array}$ & \begin{tabular}{l}
$\mathcal{I}$ \\
\multirow{0}{0}{}
\end{tabular} \\
\hline \multirow[t]{2}{*}{ Ease of transfer } & $\begin{array}{c}\text { స్ } \\
\text { - }\end{array}$ & $\begin{array}{l}\text { గై } \\
\text { o. }\end{array}$ & \begin{tabular}{l}
$\infty$ \\
\hdashline \\
\hdashline
\end{tabular} & 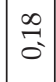 & $\begin{array}{l}\text { oे } \\
\text { á }\end{array}$ & $\begin{array}{l}\text { 㣽 } \\
\text { o }\end{array}$ & $\begin{array}{l}\hat{o} \\
\text { ô }\end{array}$ & 0 & 0 & 芯 & $\begin{array}{l}0 \\
0 \\
0 \\
0\end{array}$ & 0 & 0 & $\left|\begin{array}{l}\infty \\
\infty \\
0 \\
0 \\
0\end{array}\right|$ & 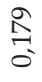 & $\mid \begin{array}{l}\overrightarrow{0} \\
\vec{\sigma}\end{array}$ & $\left|\begin{array}{c}1 \\
m \\
0 \\
0\end{array}\right|$ & 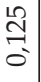 & సे & $\stackrel{0}{7}$ \\
\hline & 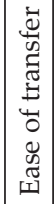 & 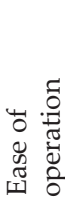 & 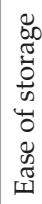 & 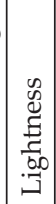 & 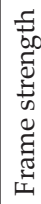 & 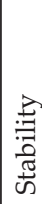 & 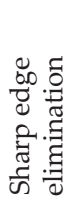 & 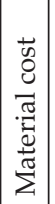 & 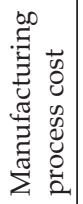 & 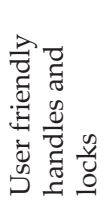 & 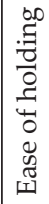 & 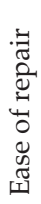 & 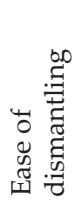 & 岂 & 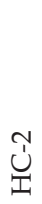 & $\begin{array}{c}0 \\
ن \\
ن\end{array}$ & 䓌 & 足 & 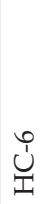 & 岁 \\
\hline
\end{tabular}




\begin{tabular}{|c|c|c|c|c|c|c|c|c|c|c|c|c|c|c|c|c|c|c|c|c|c|}
\hline HC-7 & 焉 & 옹 & $\begin{array}{l}F \\
0 \\
0\end{array}$ & $\begin{array}{l}\infty \\
0 \\
0 \\
0 \\
0\end{array}$ & 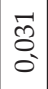 & $\approx$ & $\begin{array}{l}\mathscr{2} \\
8 \\
0 \\
0\end{array}$ & $\begin{array}{l}\text { ลิ } \\
\text { - }\end{array}$ & $\stackrel{\infty}{0}$ & $\frac{2}{3}$ & $\begin{array}{l}\text { 合 } \\
\text { O. }\end{array}$ & o̊ & $\begin{array}{l}18 \\
8 \\
0\end{array}$ & $\circ$ & 0 & 0 & 0 & 0 & 0 & 0 & $\stackrel{\circ}{\circ}$ \\
\hline HC-6 & $\stackrel{8}{8}$ & 롱 & $\begin{array}{l}F \\
0 \\
0\end{array}$ & $\begin{array}{l}0 \\
\hat{0} \\
0\end{array}$ & $\begin{array}{l}0 \\
\tilde{O} \\
0\end{array}$ & 商 & \begin{tabular}{l}
$\infty$ \\
\multirow{0}{0}{} \\
0
\end{tabular} & 疍 & $\stackrel{\infty}{\circ}$ & $\begin{array}{l}\tilde{I} \\
0\end{array}$ & $\begin{array}{l}0 \\
\stackrel{0}{0} \\
0\end{array}$ & $\begin{array}{l}0 \\
\text { ô } \\
0\end{array}$ & $\underset{0}{0}$ & 0 & 0 & 0 & 0 & 0 & 0 & 0 & $\stackrel{\circ}{\circ}$ \\
\hline HC-5 & 兽 & 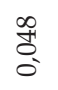 & $\begin{array}{l}F \\
8 \\
0\end{array}$ & $\begin{array}{l}\infty \\
0 \\
0 \\
0 \\
0\end{array}$ & $\begin{array}{l}1 \\
\text { है } \\
0 \\
0\end{array}$ & $\begin{array}{l}\infty \\
m \\
\tilde{m} \\
\vdots\end{array}$ & $\begin{array}{l}\hat{A} \\
\text { Oे }\end{array}$ & $\stackrel{m}{0}$ & \begin{tabular}{l}
0 \\
\multirow{2}{0}{} \\
0 \\
0
\end{tabular} & $\begin{array}{l}\text { I } \\
\stackrel{2}{0}\end{array}$ & $\begin{array}{l}0 \\
\hat{0} \\
0 \\
0\end{array}$ & 合 & $\begin{array}{l}8 \\
8 \\
0\end{array}$ & $\circ$ & 0 & 0 & 0 & 0 & 0 & 0 & $\stackrel{\circ}{\rightarrow}$ \\
\hline HC-4 & $\begin{array}{l}\infty \\
0 \\
0 \\
0 \\
0\end{array}$ & $\stackrel{10}{0}$ & $\begin{array}{c}\text { के } \\
0 \\
0\end{array}$ & $\begin{array}{ll}1 \\
0 \\
0 \\
0\end{array}$ & $\begin{array}{c}\text { ¿े } \\
\text { Oे } \\
\text { a }\end{array}$ & ב⿱ & $\begin{array}{l}\infty \\
\stackrel{\infty}{0} \\
0\end{array}$ & בิ & $\begin{array}{l}\infty \\
0 \\
0 \\
0\end{array}$ & స్ & $\begin{array}{l}0 \\
0 \\
0 \\
0\end{array}$ & $\begin{array}{l}2 \\
\delta \\
0 \\
0\end{array}$ & $\underset{8}{0}$ & 0 & 0 & 0 & 0 & 0 & 0 & 0 & $\stackrel{\circ}{\rightarrow}$ \\
\hline HC-3 & $\begin{array}{l}2 \\
8 \\
0 \\
0\end{array}$ & $\begin{array}{l}\text { fे } \\
\text { o } \\
0\end{array}$ & $\begin{array}{l}\text { Fै } \\
0\end{array}$ & $\begin{array}{l}\infty \\
0 \\
0 \\
0 \\
0\end{array}$ & $\begin{array}{l}\overrightarrow{8} \\
0 \\
0\end{array}$ & 常 & i⿱ & $\begin{array}{l}\text { I } \\
\text { - }\end{array}$ & $\stackrel{\infty}{\infty}$ & $\begin{array}{l}\hat{\Xi} \\
\text { ¿ }\end{array}$ & $\stackrel{0}{\circ}$ & 年 & $\begin{array}{l}\text { Jे } \\
0 \\
0\end{array}$ & $\circ$ & 0 & 0 & 0 & 0 & 0 & 0 & $\stackrel{\circ}{\circ}$ \\
\hline HC-2 & 畠 & \begin{tabular}{l}
$\infty$ \\
\multirow{3}{*}{} \\
0 \\
0
\end{tabular} & $\begin{array}{l}\text { H } \\
\text { O } \\
0\end{array}$ & 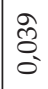 & $\begin{array}{l}\overline{8} \\
0 \\
0\end{array}$ & $\stackrel{m}{0}$ & $\begin{array}{l}\text { H } \\
0 \\
0 \\
0\end{array}$ & $\begin{array}{l}\text { लै } \\
\text { - }\end{array}$ & $\begin{array}{l}\text { L } \\
\text { S } \\
0 \\
0\end{array}$ & 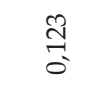 & $\begin{array}{l}\text { t } \\
\text { Oे } \\
0\end{array}$ & 落 & ஜ̊ & 0 & 0 & 0 & 0 & 0 & 0 & 0 & ํ. \\
\hline HC-1 & 8 & 옹 & $\begin{array}{l}\text { H } \\
0 \\
0\end{array}$ & $\mid \begin{array}{l}\infty \\
0 \\
0 \\
0\end{array}$ & $\stackrel{8}{8}$ & F & 营 & 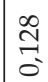 & ڤ్ & 苾 & $\stackrel{0}{0}$ & o & $\begin{array}{l}\overrightarrow{8} \\
0 \\
0\end{array}$ & 0 & 0 & 0 & 0 & 0 & o & 0 & $\stackrel{\sim}{\circ}$ \\
\hline $\begin{array}{l}\text { Ease of } \\
\text { dismantling }\end{array}$ & ồ & 0 & $\begin{array}{l}\infty \\
0 \\
0 \\
0\end{array}$ & $\begin{array}{l}0 \\
0 \\
0 \\
0\end{array}$ & 0 & 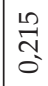 & 莒 & 0 & 0 & ก̂ & $\begin{array}{l}1 \\
0 \\
0 \\
0\end{array}$ & 0 & 0 & $\left|\begin{array}{l}1 \\
0 \\
0 \\
0\end{array}\right|$ & $\begin{array}{l}0 \\
\tilde{0} \\
0 \\
0\end{array}$ & $\begin{array}{c}8 \\
0 \\
0\end{array} \mid$ & 商 & $\mid \begin{array}{l}0 \\
0 \\
0 \\
0\end{array}$ & $\begin{array}{l}2 \\
0 \\
0 \\
0\end{array}$ & $\left|\begin{array}{c}\vec{\delta} \\
0 \\
\vdots\end{array}\right|$ & $\stackrel{\circ}{\rightarrow}$ \\
\hline Ease of repair & 0 & 照 & $\circ$ & $\begin{array}{l}0 \\
0 \\
0\end{array}$ & \begin{tabular}{l}
18 \\
\hdashline \\
0
\end{tabular} & 0 & 0 & 0 & 0 & o & 0 & 0 & 0 & $\begin{array}{l}10 \\
0 \\
0 \\
0\end{array}$ & $\begin{array}{l}\mathbb{R} \\
\hat{0} \\
0 \\
0\end{array}$ & 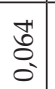 & $\mid \begin{array}{l}1 \\
0 \\
0 \\
0\end{array}$ & \begin{tabular}{|l}
0 \\
0 \\
0 \\
0
\end{tabular} & के & $\begin{array}{ll}2 \\
0 \\
0\end{array}$ & $\stackrel{\circ}{\circ}$ \\
\hline Ease of holding & 年 & 胥 & $\begin{array}{l}\infty \\
\stackrel{0}{0} \\
0\end{array}$ & 0 & $\circ$ & ت્5 & $\begin{array}{l}\text { L } \\
\text { o } \\
0\end{array}$ & 0 & 0 & 0 & 0 & 0 & 0 & $\begin{array}{l}8 \\
\vdots \\
0\end{array}$ & $\begin{array}{c}0 \\
0 \\
0 \\
0\end{array}$ & $\left|\begin{array}{c}0 \\
0 \\
0 \\
0\end{array}\right|$ & $\mid$ & $\left|\begin{array}{c}\vec{m} \\
0 \\
0\end{array}\right|$ & $\begin{array}{l}\text { है } \\
0 \\
0\end{array}$ & $\begin{array}{l}8 \\
0 \\
0\end{array}$ & $\stackrel{\circ}{-}$ \\
\hline $\begin{array}{l}\text { User friendly } \\
\text { handles and locks }\end{array}$ & 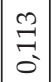 & o & $\begin{array}{l}0 \\
0 \\
0 \\
0\end{array}$ & 0 & $\circ$ & $\frac{\infty}{2}$ & 点 & $\frac{8}{4}$ & ठ̀ & o & 0 & 0 & 0 & $\left|\begin{array}{l}1 \\
0 \\
0 \\
0\end{array}\right|$ & $\begin{array}{l}0 \\
0 \\
0 \\
0\end{array}$ & $\begin{array}{c}0 \\
0 \\
0 \\
0\end{array}$ & 童 & $\left|\begin{array}{c}2 \\
0 \\
0 \\
0\end{array}\right|$ & $\begin{array}{l}\text { İ } \\
0 \\
0\end{array}$ & $\mid \begin{array}{c}\vec{\delta} \\
0\end{array}$ & $\stackrel{\circ}{\rightarrow}$ \\
\hline $\begin{array}{l}\text { Manufacturing } \\
\text { process cost }\end{array}$ & 0 & 0 & 0 & $\begin{array}{l}\text { से } \\
\text { लू } \\
0\end{array}$ & 总 & 0 & 0 & 0 & 0 & 0 & 0 & 0 & 0 & $\begin{array}{l}\infty \\
0 \\
0 \\
0\end{array}$ & $\begin{array}{l}5 \\
\text { S } \\
0\end{array}$ & $\left|\begin{array}{c}5 \\
0 \\
0 \\
0\end{array}\right|$ & $\mid \begin{array}{l}10 \\
8 \\
0 \\
0\end{array}$ & $\begin{array}{l}1 \\
0 \\
0 \\
0\end{array}$ & 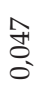 & $\left|\begin{array}{l}\infty \\
0 \\
0 \\
0 \\
0\end{array}\right|$ & $\stackrel{\circ}{-}$ \\
\hline Material cost & 0 & 0 & 0 & 商 & $\begin{array}{l}\text { ô } \\
\text { â } \\
0\end{array}$ & 0 & 0 & 0 & 0 & 0 & 0 & 0 & 0 & $\begin{array}{l}0 \\
\vdots \\
0 \\
0\end{array}$ & $\begin{array}{l}0 \\
\vdots \\
0 \\
0\end{array}$ & 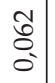 & 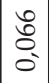 & $\begin{array}{l}0 \\
0 \\
0 \\
0\end{array}$ & $\begin{array}{l}\infty \\
\text { के } \\
0 \\
0\end{array}$ & $\mid \begin{array}{l}0 \\
0 \\
0 \\
0\end{array}$ & $\stackrel{\circ}{\rightarrow}$ \\
\hline $\begin{array}{l}\text { Sharp edge } \\
\text { elimination }\end{array}$ & $\begin{array}{l}+1 \\
\text { 孛 } \\
0 \\
0\end{array}$ & $\begin{array}{l}\hat{0} \\
\text { 乃े } \\
0\end{array}$ & ה్ & 0 & 0 & 0 & 0 & 0 & 0 & $\overrightarrow{\tilde{\sigma}}$ & ¿ & 命 & 产 & $\begin{array}{l}0 \\
8 \\
0 \\
0\end{array}$ & $\begin{array}{l}\text { o. } \\
\vdots \\
0\end{array}$ & $\mid \begin{array}{c}1 \\
\delta \\
0 \\
0\end{array}$ & $\mid \begin{array}{c}1 \\
\delta \\
0 \\
0\end{array}$ & $\begin{array}{c}1 \\
\delta \\
0 \\
0\end{array}$ & 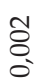 & $\left|\begin{array}{c}1 \\
\delta \\
0 \\
0\end{array}\right|$ & $\stackrel{\circ}{-}$ \\
\hline Stability & 鸽 & \& & $\begin{array}{l}\ddot{1} \\
0 \\
0\end{array}$ & 0 & $\begin{array}{l}\pi \\
8 \\
0\end{array}$ & 勇 & 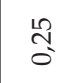 & 0 & 0 & 0 & 0 & 0 & 0 & 0 & 0 & 0 & 0 & 0 & 0 & 0 & 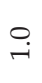 \\
\hline Frame strengh & $\mid \begin{array}{l}\infty \\
\infty \\
0 \\
0\end{array}$ & 8 & 音 & 咅 & $\begin{array}{ll}1 \\
0 \\
0 \\
0\end{array}$ & 0 & 0 & סे & $\stackrel{\substack{m \\
\hdashline}}{\stackrel{0}{0}}$ & o & 0 & 0 & 0 & $\begin{array}{l}\stackrel{2}{2} \\
0 \\
0\end{array}$ & $\begin{array}{l}\text { के } \\
0 \\
0\end{array}$ & $\mid \begin{array}{l}1 \\
0 \\
0 \\
0\end{array}$ & F & $\begin{array}{l}8 \\
0 \\
0\end{array}$ & $\begin{array}{l}\text { ¿े } \\
\text { ठे }\end{array}$ & $\mid \begin{array}{l}0 \\
0 \\
0 \\
0\end{array}$ & $\stackrel{\circ}{\rightarrow}$ \\
\hline Lightness & $\begin{array}{l}N \\
\hat{\delta} \\
0\end{array}$ & $\begin{array}{l}5 \\
\text { S } \\
0\end{array}$ & $\begin{array}{l}\text { 岁 } \\
\text { o. }\end{array}$ & $\begin{array}{l}12 \\
20 \\
0 \\
0\end{array}$ & $\begin{array}{l}1 \\
\text { ถి } \\
0 \\
0\end{array}$ & 0 & 0 & $\begin{array}{l}3 \\
\stackrel{0}{0} \\
\stackrel{-}{0}\end{array}$ & $\vec{\sigma}$ & o & 0 & $\begin{array}{l}1 \\
0 \\
0 \\
0\end{array}$ & \&̊ & $\begin{array}{l}3 \\
8 \\
0 \\
0\end{array}$ & $\begin{array}{l}+ \\
0 \\
0\end{array}$ & $\left|\begin{array}{l}1 \\
0 \\
0 \\
0\end{array}\right|$ & $\mid \begin{array}{l}H \\
0 \\
0 \\
0\end{array}$ & 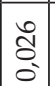 & $\begin{array}{l}2 \\
0 \\
0\end{array}$ & $\mid \begin{array}{l}0 \\
0 \\
0 \\
0\end{array}$ & $\stackrel{\circ}{\rightarrow}$ \\
\hline Ease of storage & $\begin{array}{l}\infty \\
\infty \\
0 \\
0\end{array}$ & $\begin{array}{l}\infty \\
\stackrel{\infty}{0} \\
0 \\
0\end{array}$ & $\begin{array}{l}0 \\
\text { ठे } \\
0\end{array}$ & $\begin{array}{l}0 \\
0 \\
0 \\
0\end{array}$ & $\begin{array}{l}0 \\
8 \\
0 \\
0\end{array}$ & 兽 & ठ̊ & o & 0 & 0 & 0 & 0 & o & $\begin{array}{l}\infty \\
\ddot{8} \\
0 \\
0\end{array}$ & 영 & $\mid \begin{array}{c}\overrightarrow{1} \\
0 \\
0\end{array}$ & $\begin{array}{l}0 \\
0 \\
0 \\
0\end{array}$ & $\mid \begin{array}{l}0 \\
0 \\
0 \\
0\end{array}$ & $\begin{array}{l}\infty \\
0 \\
0 \\
0\end{array}$ & 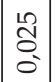 & $\stackrel{\circ}{\rightarrow}$ \\
\hline Ease of operation & $\begin{array}{l}\text { 80 } \\
8 \\
0\end{array}$ & $\stackrel{10}{8}$ & $\begin{array}{l}\sqrt{10} \\
0 \\
0\end{array}$ & 菅 & $\begin{array}{l}\text { q } \\
0 \\
0\end{array}$ & $\frac{2}{2}$ & 点 & 0 & 0 & 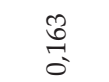 & Sิ & 0 & o & $\begin{array}{l}\infty \\
0 \\
0 \\
0\end{array}$ & $\begin{array}{l}8 \\
8 \\
0 \\
0\end{array}$ & $\begin{array}{l}0 \\
0 \\
0\end{array} \mid$ & $\left|\begin{array}{l}0 \\
0 \\
0 \\
0 \\
0\end{array}\right|$ & $\left|\begin{array}{c}0 \\
\delta \\
0 \\
0\end{array}\right|$ & $\begin{array}{l}\text { İ } \\
0\end{array}$ & $\mid \begin{array}{c}\pi \\
0 \\
0\end{array}$ & $\stackrel{\circ}{\rightarrow}$ \\
\hline \multirow[t]{2}{*}{ Ease of transfer } & \begin{tabular}{l}
\multirow{Z}{*}{} \\
8 \\
0
\end{tabular} & $\begin{array}{l}10 \\
8 \\
0 \\
0\end{array}$ & $\begin{array}{l}\text { గ̂. } \\
\text { Oे }\end{array}$ & 旁 & $\begin{array}{l}\text { fồ } \\
\text { O. }\end{array}$ & 胥 & 총 & 0 & 0 & $\begin{array}{l}3 \\
0 \\
0\end{array}$ & 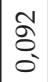 & 0 & 0 & $\begin{array}{l}\hat{⿵} \\
0 \\
0\end{array}$ & 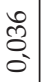 & 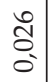 & 命 & 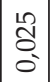 & 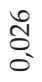 & 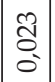 & . \\
\hline & 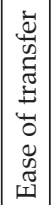 & 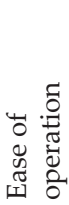 & 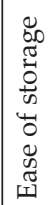 & 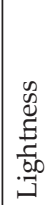 & 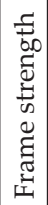 & 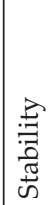 & 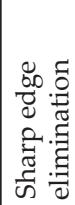 & 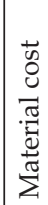 & 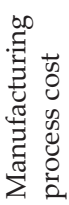 & 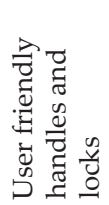 & 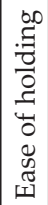 & 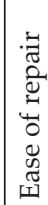 & 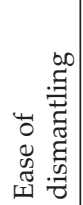 & 岂 & ن̃ & 岂 & $\bigcup_{1}^{J}$ & 足 & 茎 & $\begin{array}{l}0 \\
0 \\
\text { İ }\end{array}$ & $\sum_{\infty}$ \\
\hline
\end{tabular}




\begin{tabular}{|c|c|c|c|c|c|c|c|c|c|c|c|c|c|c|c|c|c|c|c|c|}
\hline HC-7 & $\begin{array}{l}\infty \\
0 \\
0\end{array}$ & $\begin{array}{l}\text { 点 } \\
\text { O } \\
0\end{array}$ & $\begin{array}{l}\overrightarrow{8} \\
\vdots \\
0\end{array}$ & $\begin{array}{l}19 \\
0 \\
0 \\
0\end{array}$ & $\begin{array}{l}F \\
0 \\
0\end{array}$ & $\delta^{2}$ & $\begin{array}{l}\stackrel{\circ}{0} \\
\stackrel{0}{0}\end{array}$ & $\mid \begin{array}{l}\infty \\
0 \\
0 \\
0\end{array}$ & $\begin{array}{l}\stackrel{1}{2} \\
\stackrel{\sigma}{0} \\
0\end{array}$ & $\begin{array}{l}\mathbb{H} \\
\text { O } \\
0\end{array}$ & $\begin{array}{l}0 \\
0 \\
0 \\
0\end{array}$ & $\left|\begin{array}{l}\infty \\
0 \\
0 \\
0\end{array}\right|$ & 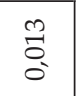 & $\begin{array}{l}\text { त̃ } \\
\text { Oे }\end{array}$ & $\begin{array}{l}\bar{\delta} \\
\delta \\
\delta\end{array}$ & $\begin{array}{l}2 \\
\overrightarrow{0} \\
0 \\
0\end{array}$ & $\begin{array}{l}\infty \\
\vdots \\
0 \\
0\end{array}$ & \begin{tabular}{|l|l}
12 \\
0 \\
0 \\
0
\end{tabular} & $\mid \begin{array}{l}+ \\
0 \\
0 \\
0\end{array}$ & $\begin{array}{l}m \\
0 \\
0 \\
0\end{array}$ \\
\hline HC-6 & $\begin{array}{l}\mathscr{0} \\
0 \\
0 \\
0\end{array}$ & $\begin{array}{l}\text { 点 } \\
\text { O } \\
0\end{array}$ & $\begin{array}{l}\vec{b} \\
0 \\
0\end{array}$ & $\begin{array}{l}\frac{18}{8} \\
0 \\
0\end{array}$ & $\begin{array}{l}\text { F } \\
0 \\
0\end{array}$ & 3 & $\begin{array}{l}\stackrel{8}{\circ} \\
\text { : }\end{array}$ & $\left|\begin{array}{l}\infty \\
\delta \\
\delta \\
0\end{array}\right|$ & 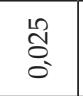 & $\begin{array}{l}\text { H্ } \\
\text { Oे } \\
0\end{array}$ & 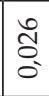 & $\mid \begin{array}{l}\infty \\
\vdots \\
0 \\
0\end{array}$ & $\begin{array}{l}0 \\
0 \\
0 \\
0\end{array}$ & $\begin{array}{l}\mathbb{Z} \\
\text { d. } \\
\text { - }\end{array}$ & $\overrightarrow{\widetilde{\delta}}$ & $\mid \begin{array}{l}2 \\
\bar{\sigma} \\
0\end{array}$ & $\begin{array}{l}\infty \\
\vdots \\
0 \\
0\end{array}$ & $\mid$\begin{tabular}{|l}
10 \\
0 \\
0 \\
0
\end{tabular} & 营 & $\stackrel{m}{a}$ \\
\hline HC-5 & $\begin{array}{l}\infty \\
0 \\
0 \\
0\end{array}$ & $\begin{array}{l}\text { No } \\
\text { O. } \\
0\end{array}$ & $\begin{array}{l}\overline{8} \\
0\end{array}$ & $\begin{array}{l}\frac{2}{4} \\
0 \\
0\end{array}$ & $\begin{array}{l}F \\
8 \\
0\end{array}$ & n. & $\begin{array}{l}\stackrel{a}{0} \\
\stackrel{5}{0}\end{array}$ & $\mid \begin{array}{l}\infty \\
0 \\
0 \\
0 \\
0\end{array}$ & $\begin{array}{l}\stackrel{L}{\Omega} \\
\stackrel{0}{0} \\
0\end{array}$ & \begin{tabular}{l}
\multirow{H}{*}{} \\
$\stackrel{0}{\circ}$
\end{tabular} & 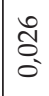 & $\mid \begin{array}{l}\infty \\
\vdots \\
0 \\
0\end{array}$ & $\begin{array}{l}0 \\
0 \\
0 \\
0\end{array}$ & $\begin{array}{l}\stackrel{\Delta}{0} \\
\text { Oे }\end{array}$ & $\begin{array}{l}\bar{\delta} \\
\delta \\
\delta\end{array}$ & $\begin{array}{l}\hat{2} \\
\bar{\sigma} \\
0\end{array}$ & $\begin{array}{l}\infty \\
\vdots \\
0 \\
0\end{array}$ & \begin{tabular}{|l|l}
12 \\
0 \\
0 \\
0
\end{tabular} & $\begin{array}{l}\vec{H} \\
0 \\
0 \\
0\end{array}$ & ह̂ \\
\hline HC-4 & $\begin{array}{l}\infty \\
0 \\
0 \\
0\end{array}$ & $\begin{array}{l}\text { Ln } \\
\text { On } \\
0\end{array}$ & $\begin{array}{l}\vec{b} \\
0 \\
0\end{array}$ & $\begin{array}{l}18 \\
0 \\
0\end{array}$ & $\begin{array}{l}F \\
0 \\
0\end{array}$ & $\overbrace{0}^{2}$ & $\begin{array}{l}\stackrel{8}{0} \\
\stackrel{0}{0}\end{array}$ & $\mid \begin{array}{l}\infty \\
0 \\
0 \\
0 \\
0\end{array}$ & $\begin{array}{l}\stackrel{L}{\mathrm{~S}} \\
\mathrm{O}\end{array}$ & $\begin{array}{l}\text { H } \\
\text { o } \\
0\end{array}$ & 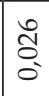 & $\mid \begin{array}{l}\infty \\
0 \\
0 \\
0\end{array}$ & $\begin{array}{l}0 \\
0 \\
0 \\
0\end{array}$ & $\begin{array}{l}\mathbb{d} \\
\text { d. } \\
\text { - }\end{array}$ & 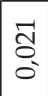 & $\begin{array}{l}\hat{\sigma} \\
\overrightarrow{0} \\
0\end{array}$ & $\begin{array}{l}\infty \\
\vdots \\
0 \\
0\end{array}$ & \begin{tabular}{|c|}
12 \\
0 \\
0 \\
0
\end{tabular} & $\begin{array}{l}\vec{J} \\
0 \\
0\end{array}$ & $\stackrel{m}{a}$ \\
\hline $\mathrm{HC}-3$ & $\begin{array}{l}\text { D } \\
0 \\
0 \\
0\end{array}$ & $\begin{array}{l}\stackrel{L}{2} \\
\text { O. } \\
0\end{array}$ & $\begin{array}{l}\vec{b} \\
0 \\
0\end{array}$ & $\begin{array}{l}19 \\
0 \\
0\end{array}$ & 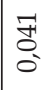 & 3. & $\begin{array}{l}\stackrel{\circ}{0} \\
\stackrel{0}{0}\end{array}$ & $\mid \begin{array}{l}\infty \\
0 \\
0 \\
0 \\
0\end{array}$ & $\begin{array}{l}\stackrel{L}{\mathrm{~S}} \\
\stackrel{\mathrm{O}}{0}\end{array}$ & $\begin{array}{l}\text { H } \\
\text { o } \\
0\end{array}$ & 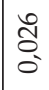 & $\mid \begin{array}{l}\infty \\
\vdots \\
0 \\
0\end{array}$ & $\begin{array}{l}0 \\
0 \\
0 \\
0\end{array}$ & $\begin{array}{l}\text { त゙ } \\
\text { Oे }\end{array}$ & స్ & $\begin{array}{l}\hat{\sigma} \\
\tilde{\sigma} \\
0\end{array}$ & $\begin{array}{l}\infty \\
\vdots \\
0 \\
0\end{array}$ & \begin{tabular}{|l}
10 \\
0 \\
0 \\
0
\end{tabular} & $\begin{array}{l}\text { 岁 } \\
0 \\
0\end{array}$ & $\stackrel{m}{a}$ \\
\hline HC-2 & $\begin{array}{l}\infty \\
0 \\
0 \\
0\end{array}$ & $\begin{array}{l}\text { na } \\
\text { O. } \\
0\end{array}$ & $\begin{array}{l}\overrightarrow{8} \\
0\end{array}$ & 告 & F & mo & $\begin{array}{l}\stackrel{\circ}{\circ} \\
\stackrel{0}{0}\end{array}$ & $\mid \begin{array}{l}\infty \\
\delta \\
\delta \\
0\end{array}$ & 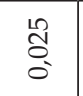 & $\begin{array}{l}\stackrel{+}{*} \\
0 \\
0\end{array}$ & 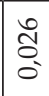 & $\mid \begin{array}{l}\infty \\
\vdots \\
0 \\
0\end{array}$ & $\begin{array}{l}0 \\
0 \\
0 \\
0\end{array}$ & $\begin{array}{l}\text { ḋ } \\
\text { Oे }\end{array}$ & $\begin{array}{l}\widetilde{\Xi} \\
\text { o }\end{array}$ & $\begin{array}{l}\hat{\sigma} \\
\tilde{\sigma} \\
0\end{array}$ & $\mid \begin{array}{l}\infty \\
\vdots \\
0 \\
0\end{array}$ & \begin{tabular}{|l}
10 \\
0 \\
0 \\
0
\end{tabular} & $\begin{array}{l}+ \\
0 \\
0 \\
0\end{array}$ & a \\
\hline HC-1 & $\begin{array}{l}\infty \\
0 \\
0 \\
0\end{array}$ & $\begin{array}{l}\text { L } \\
\text { O. } \\
0\end{array}$ & $\begin{array}{l}\overrightarrow{8} \\
\stackrel{0}{\circ}\end{array}$ & $\begin{array}{l}19 \\
0 \\
0\end{array}$ & F & $\stackrel{2}{0}$ & $\begin{array}{l}\text { oे } \\
\stackrel{0}{0}\end{array}$ & $\mid \begin{array}{l}\infty \\
0 \\
0 \\
0\end{array}$ & 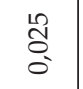 & $\begin{array}{l}\text { H } \\
\text { o } \\
0\end{array}$ & 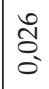 & $\mid \begin{array}{l}\infty \\
\vdots \\
0 \\
0\end{array}$ & $\begin{array}{l}0 \\
0 \\
0 \\
0\end{array}$ & $\begin{array}{l}\mathbb{Z} \\
\text { d. } \\
\text { - }\end{array}$ & స్ & $\mid \begin{array}{l}\hat{\sigma} \\
\tilde{\sigma} \\
0\end{array}$ & $\begin{array}{l}\infty \\
\vdots \\
0 \\
0\end{array}$ & \begin{tabular}{|c|}
2 \\
0 \\
0 \\
0
\end{tabular} & $\mid \begin{array}{l}H \\
0 \\
0 \\
0\end{array}$ & $\stackrel{m}{\stackrel{2}{0}}$ \\
\hline Ease of dismantling & $\begin{array}{l}\infty \\
0 \\
0 \\
0\end{array}$ & $\begin{array}{l}\text { L } \\
\text { 足 } \\
0\end{array}$ & $\begin{array}{l}\vec{b} \\
:\end{array}$ & $\begin{array}{l}18 \\
0 \\
0\end{array}$ & $\begin{array}{l}\text { F } \\
0 \\
0\end{array}$ & $\sigma_{0}^{2}$ & $\begin{array}{l}\text { oे } \\
\text { - }\end{array}$ & $\mid \begin{array}{l}\infty \\
0 \\
0 \\
0 \\
0\end{array}$ & 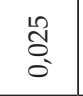 & $\begin{array}{l}\mathbb{H} \\
\text { Oे } \\
0\end{array}$ & 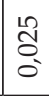 & $\mid \begin{array}{l}\infty \\
\vdots \\
0 \\
0\end{array}$ & $\begin{array}{l}0 \\
0 \\
0 \\
0\end{array}$ & 范 & $\begin{array}{l}\bar{\delta} \\
\delta\end{array}$ & $\begin{array}{l}\hat{\sigma} \\
\tilde{0} \\
0\end{array}$ & $\begin{array}{l}\hat{0} \\
0 \\
0\end{array}$ & \begin{tabular}{|l}
10 \\
0 \\
0 \\
0
\end{tabular} & $\begin{array}{l}\vec{H} \\
0 \\
0 \\
0\end{array}$ & and \\
\hline Ease of repair & $\begin{array}{l}\mathscr{0} \\
0 \\
0 \\
0\end{array}$ & $\begin{array}{l}\stackrel{\mathbb{L}}{0} \\
\text { O. } \\
0\end{array}$ & $\begin{array}{l}\vec{b} \\
0 \\
0\end{array}$ & $\begin{array}{l}0 \\
0 \\
0 \\
0\end{array}$ & $\begin{array}{l}\text { F } \\
0 \\
0\end{array}$ & $\begin{array}{l}\text { ते } \\
\text { तू }\end{array}$ & $\begin{array}{l}\stackrel{\infty}{0} \\
\stackrel{0}{0}\end{array}$ & $\mid \begin{array}{l}\infty \\
0 \\
0 \\
0 \\
0\end{array}$ & $\begin{array}{l}\stackrel{L}{Q} \\
\stackrel{0}{0} \\
0\end{array}$ & 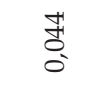 & $\begin{array}{l}0 \\
\delta \\
0 \\
0\end{array}$ & $\mid \begin{array}{l}\infty \\
\vdots \\
0 \\
0\end{array}$ & $\begin{array}{l}m \\
0 \\
0\end{array}$ & 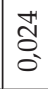 & స్ & $\begin{array}{l}\hat{\sigma} \\
\tilde{\sigma} \\
0\end{array}$ & $\begin{array}{l}\infty \\
\vdots \\
0 \\
0\end{array}$ & \begin{tabular}{|l|l}
10 \\
0 \\
0 \\
0
\end{tabular} & 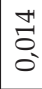 & a \\
\hline Ease of holding & $\begin{array}{l}\infty \\
0 \\
0 \\
0\end{array}$ & $\begin{array}{l}\text { Ln } \\
\text { L } \\
0 \\
0\end{array}$ & $\begin{array}{l}\overrightarrow{6} \\
0 \\
0\end{array}$ & $\begin{array}{l}19 \\
0 \\
0 \\
0\end{array}$ & $\begin{array}{l}F \\
0 \\
0\end{array}$ & 8 & $\begin{array}{l}\stackrel{8}{0} \\
\stackrel{0}{0}\end{array}$ & $\mid \begin{array}{l}\infty \\
0 \\
0 \\
0 \\
0\end{array}$ & 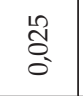 & \begin{tabular}{l}
\multirow{H}{*}{} \\
O
\end{tabular} & \begin{tabular}{|l}
$\stackrel{2}{0}$ \\
0 \\
0
\end{tabular} & $\mid \begin{array}{l}\infty \\
0 \\
0 \\
0\end{array}$ & $\begin{array}{l}m \\
\stackrel{2}{0} \\
0\end{array}$ & $\begin{array}{l}\mathbb{J} \\
\text { Oे }\end{array}$ & $\overrightarrow{\tilde{\delta}}$ & $\begin{array}{l}\hat{\sigma} \\
0 \\
0\end{array}$ & 응 & $\mid \begin{array}{l}10 \\
0 \\
0 \\
0\end{array}$ & $\begin{array}{l}\overrightarrow{0} \\
\stackrel{5}{0} \\
0\end{array}$ & $\frac{m}{a}$ \\
\hline $\begin{array}{l}\text { User friendly handles } \\
\text { and locks }\end{array}$ & $\begin{array}{l}\infty \\
\infty \\
0 \\
0\end{array}$ & $\begin{array}{l}\text { L } \\
\text { S } \\
0 \\
0\end{array}$ & $\begin{array}{l}\overline{6} \\
\vdots \\
0\end{array}$ & $\begin{array}{l}19 \\
0 \\
0 \\
0\end{array}$ & $\begin{array}{l}\text { F } \\
0 \\
0\end{array}$ & m & $\begin{array}{l}\stackrel{8}{0} \\
\vdots \\
0\end{array}$ & $\left|\begin{array}{l}\infty \\
\infty \\
0 \\
0\end{array}\right|$ & 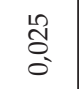 & $\begin{array}{l}\text { H } \\
\text { \& } \\
0\end{array}$ & 离 & $\left|\begin{array}{l}\infty \\
\vdots \\
0 \\
0\end{array}\right|$ & $\begin{array}{l}m \\
\vdots \\
0\end{array}$ & 芯 & స్ & $\begin{array}{l}2 \\
\bar{\delta} \\
0 \\
0\end{array}$ & 会 & \begin{tabular}{|l|}
12 \\
0 \\
0 \\
0
\end{tabular} & 章 & co \\
\hline $\begin{array}{l}\text { Manufacturing } \\
\text { process cost }\end{array}$ & $\begin{array}{l}\infty \\
\infty \\
0 \\
0\end{array}$ & $\begin{array}{l}12 \\
\text { L } \\
0 \\
0\end{array}$ & $\begin{array}{l}\overrightarrow{8} \\
\stackrel{-}{\circ}\end{array}$ & 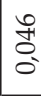 & $\begin{array}{l}\text { F } \\
0 \\
0\end{array}$ & 离 & $\begin{array}{l}\infty \\
\stackrel{\infty}{0} \\
0\end{array}$ & $\begin{array}{l}\infty \\
\infty \\
0 \\
0 \\
0\end{array}$ & $\stackrel{2}{0}$ & $\begin{array}{l}\text { Hi } \\
\text { O }\end{array}$ & 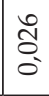 & $\mid \begin{array}{l}\infty \\
0 \\
\vdots \\
0 \\
0\end{array}$ & $\begin{array}{l}m \\
\stackrel{3}{0} \\
0\end{array}$ & 总 & 帘 & $\begin{array}{l}2 \\
\bar{\delta} \\
0 \\
0\end{array}$ & $\begin{array}{l}\infty \\
0 \\
\vdots \\
0 \\
0\end{array}$ & \begin{tabular}{|l|}
10 \\
0 \\
0 \\
0
\end{tabular} & $\begin{array}{l}0 \\
0 \\
0 \\
0\end{array}$ & $\frac{m}{c}$ \\
\hline Material cost & $\begin{array}{l}\infty \\
\infty \\
0 \\
0\end{array}$ & $\begin{array}{l}12 \\
\text { 点 } \\
0\end{array}$ & 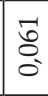 & $\begin{array}{l}0 \\
0 \\
0 \\
0\end{array}$ & $\begin{array}{l}\text { F } \\
0 \\
0\end{array}$ & సू. & $\begin{array}{l}\infty \\
\stackrel{\infty}{0} \\
0\end{array}$ & $\begin{array}{l}\infty \\
0 \\
0 \\
0 \\
0\end{array}$ & 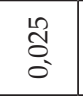 & 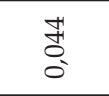 & 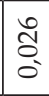 & $\mid \begin{array}{l}\infty \\
0 \\
0 \\
0\end{array}$ & $\begin{array}{l}m \\
\stackrel{2}{0} \\
0\end{array}$ & $\begin{array}{l}\text { ते } \\
\text { Oे }\end{array}$ & స్ & $\begin{array}{l}2 \\
\vdots \\
0 \\
0\end{array}$ & $\begin{array}{l}\infty \\
\vdots \\
0 \\
0\end{array}$ & \begin{tabular}{|l|}
10 \\
0 \\
0 \\
0
\end{tabular} & 章 & $\stackrel{9}{2}$ \\
\hline $\begin{array}{l}\text { Sharp edge } \\
\text { elimination }\end{array}$ & $\begin{array}{l}\infty \\
\infty \\
0 \\
0\end{array}$ & $\begin{array}{l}\text { Ln } \\
\text { 今 } \\
0 \\
0\end{array}$ & $\begin{array}{l}\text { है } \\
\stackrel{0}{\circ}\end{array}$ & $\begin{array}{l}\text { L } \\
0 \\
0 \\
0\end{array}$ & $\begin{array}{l}\text { F } \\
0 \\
0\end{array}$ & $a_{0}^{2}$ & $\begin{array}{l}\text { oे } \\
\text { : }\end{array}$ & $\mid \begin{array}{l}\infty \\
0 \\
0 \\
0 \\
0\end{array}$ & $\begin{array}{l}\stackrel{1}{\Omega} \\
\stackrel{0}{0}\end{array}$ & \begin{tabular}{l}
\multirow{2}{*}{} \\
$\stackrel{0}{\circ}$
\end{tabular} & $\begin{array}{l}0 \\
\delta \\
0 \\
0\end{array}$ & $\mid \begin{array}{l}\infty \\
0 \\
0 \\
0\end{array}$ & $\begin{array}{l}m \\
\stackrel{2}{0} \\
0\end{array}$ & 离 & ్ㅗ & $\begin{array}{l}\hat{\sigma} \\
\tilde{0} \\
0\end{array}$ & $\begin{array}{l}\hat{a} \\
\vdots \\
0\end{array}$ & \begin{tabular}{|l}
10 \\
0 \\
0 \\
0
\end{tabular} & $\begin{array}{l}\vec{J} \\
0 \\
0\end{array}$ & $\stackrel{m}{a}$ \\
\hline Stability & $\begin{array}{l}\infty \\
0 \\
0 \\
0\end{array}$ & $\begin{array}{l}\text { In } \\
\text { 今. } \\
0\end{array}$ & $\begin{array}{l}\overrightarrow{8} \\
\stackrel{0}{\circ}\end{array}$ & $\begin{array}{l}19 \\
0 \\
0\end{array}$ & $\begin{array}{l}+ \\
0 \\
0\end{array}$ & $\begin{array}{l}\tilde{5} \\
\tilde{s} \\
0\end{array}$ & $\begin{array}{l}\stackrel{8}{0} \\
\stackrel{0}{0}\end{array}$ & $\mid \begin{array}{l}\infty \\
0 \\
0 \\
0 \\
0\end{array}$ & 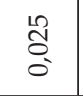 & $\begin{array}{l}\text { \& } \\
\text { ᄋ }\end{array}$ & 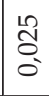 & $\begin{array}{l}0 \\
\vdots \\
0 \\
0\end{array}$ & $\begin{array}{l}m \\
\vdots \\
0 \\
0\end{array}$ & 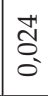 & ్ㅗ & $\begin{array}{l}a \\
\vec{\sigma} \\
0\end{array}$ & $\begin{array}{l}0 \\
0 \\
0\end{array}$ & $\begin{array}{l}\vec{a} \\
0 \\
0 \\
0\end{array}$ & $\frac{H}{0}$ & $\stackrel{m}{\stackrel{2}{0}}$ \\
\hline Frame strength & $\begin{array}{l}\infty \\
0 \\
0 \\
0\end{array}$ & $\begin{array}{l}\text { L } \\
\text { Oे } \\
0\end{array}$ & $\begin{array}{l}\overrightarrow{8} \\
\stackrel{0}{\circ}\end{array}$ & $\begin{array}{l}0 \\
0 \\
0 \\
0\end{array}$ & F & $\begin{array}{l}\text { ते } \\
\text { సू }\end{array}$ & $\begin{array}{l}\infty \\
\stackrel{0}{0} \\
0\end{array}$ & $\mid \begin{array}{l}\infty \\
\tilde{\delta} \\
0 \\
0\end{array}$ & 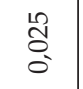 & $\begin{array}{l}\mathbb{H} \\
\stackrel{8}{0} \\
0\end{array}$ & ڤ్ & $\mid \begin{array}{l}\infty \\
\vdots \\
0 \\
0\end{array}$ & $\begin{array}{l}\stackrel{m}{0} \\
\stackrel{0}{0}\end{array}$ & 感 & 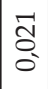 & $\begin{array}{l}\hat{\sigma} \\
\overline{0} \\
0\end{array}$ & $\begin{array}{l}\infty \\
\vdots \\
0 \\
0\end{array}$ & $\mid$\begin{tabular}{|c|}
20 \\
0 \\
0 \\
0
\end{tabular} & $\mid \begin{array}{l}\overrightarrow{1} \\
0 \\
0 \\
0\end{array}$ & $\begin{array}{l}m \\
0 \\
0\end{array}$ \\
\hline Lightness & $\begin{array}{l}\infty \\
0 \\
0 \\
0\end{array}$ & $\begin{array}{l}\text { L } \\
\text { O⿱ } \\
0 \\
0\end{array}$ & $\begin{array}{l}\vec{b} \\
0 \\
0\end{array}$ & $\begin{array}{l}0 \\
0 \\
0 \\
0\end{array}$ & $\begin{array}{l}F \\
0 \\
0\end{array}$ & $\begin{array}{l}\text { हू } \\
\text { 亏ू }\end{array}$ & $\begin{array}{l}\infty \\
\stackrel{0}{0} \\
0\end{array}$ & $\mid \begin{array}{l}\infty \\
\delta \\
\delta \\
0 \\
0\end{array}$ & $\begin{array}{l}\stackrel{L}{0} \\
\stackrel{0}{0}\end{array}$ & \begin{tabular}{l}
\multirow{8}{*}{} \\
0 \\
0
\end{tabular} & $\begin{array}{l}0 \\
\text { s. } \\
0\end{array}$ & $\mid \begin{array}{l}\infty \\
\vdots \\
0 \\
0\end{array}$ & $\begin{array}{l}0 \\
0 \\
0 \\
0\end{array}$ & 感 & ్ㅗ & $\mid \begin{array}{l}2 \\
\bar{\sigma} \\
0\end{array}$ & $\begin{array}{l}\infty \\
\vdots \\
0 \\
0\end{array}$ & $\mid \begin{array}{ll}10 \\
\vdots \\
0 \\
0\end{array}$ & $\begin{array}{l}+ \\
0 \\
0 \\
0\end{array}$ & a \\
\hline Ease of storage & $\begin{array}{l}\infty \\
0 \\
0 \\
0\end{array}$ & $\begin{array}{l}\text { In } \\
\text { 今 } \\
0 \\
0\end{array}$ & $\begin{array}{l}\overrightarrow{8} \\
\stackrel{0}{\circ}\end{array}$ & $\begin{array}{l}\text { L } \\
0 \\
0 \\
0\end{array}$ & F & $\sigma^{m}$ & $\begin{array}{l}\text { oे } \\
\stackrel{1}{0}\end{array}$ & $\mid \begin{array}{l}\infty \\
0 \\
0 \\
0 \\
0\end{array}$ & $\begin{array}{l}\stackrel{2}{0} \\
\stackrel{0}{0}\end{array}$ & \begin{tabular}{l}
\multirow{H}{*}{} \\
O
\end{tabular} & 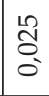 & $\mid \begin{array}{l}\infty \\
\vdots \\
0 \\
0\end{array}$ & $\begin{array}{l}\stackrel{m}{0} \\
\stackrel{0}{0}\end{array}$ & $\mid \begin{array}{l}\text { d } \\
\text { d } \\
0\end{array}$ & $\begin{array}{l}\widetilde{\Xi} \\
\text { o }\end{array}$ & $\begin{array}{l}\hat{\sigma} \\
\tilde{0} \\
0\end{array}$ & $\begin{array}{l}\hat{a} \\
\vdots \\
0\end{array}$ & \begin{tabular}{|l|l}
10 \\
0 \\
0 \\
0
\end{tabular} & 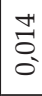 & $\stackrel{M}{0}$ \\
\hline Ease of operation & $\begin{array}{l}\infty \\
0 \\
0 \\
0\end{array}$ & $\begin{array}{l}\text { Ln } \\
\text { On } \\
0\end{array}$ & $\begin{array}{l}\overrightarrow{8} \\
\stackrel{0}{\circ}\end{array}$ & $\begin{array}{l}18 \\
0 \\
0\end{array}$ & F & $\sigma^{3}$ & $\begin{array}{l}\stackrel{\circ}{0} \\
\stackrel{\circ}{0}\end{array}$ & $\mid \begin{array}{l}\infty \\
0 \\
0 \\
0 \\
0\end{array}$ & $\begin{array}{l}\stackrel{L}{\Omega} \\
\stackrel{0}{0}\end{array}$ & \begin{tabular}{l}
\multirow{H}{*}{} \\
8
\end{tabular} & 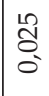 & $\mid \begin{array}{l}\infty \\
\vdots \\
0 \\
0\end{array}$ & $\begin{array}{l}m \\
\stackrel{2}{0} \\
0\end{array}$ & 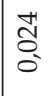 & $\overrightarrow{\widetilde{\delta}}$ & $\begin{array}{l}2 \\
\vec{\vdots} \\
0 \\
0\end{array}$ & 응 & \begin{tabular}{|l|l}
10 \\
0 \\
0 \\
0
\end{tabular} & $\mid \begin{array}{l}+ \\
0 \\
0 \\
0\end{array}$ & $\stackrel{m}{a}$ \\
\hline \multirow[t]{2}{*}{ Ease of transfer } & $\begin{array}{l}\infty \\
0 \\
0 \\
0\end{array}$ & $\begin{array}{l}\text { L } \\
\text { 号 } \\
0\end{array}$ & $\begin{array}{l}\overrightarrow{8} \\
\stackrel{0}{\circ}\end{array}$ & $\begin{array}{l}\text { Lq } \\
0 \\
0\end{array}$ & F & $\delta^{2}$ & $\begin{array}{l}\text { Oे } \\
\text { : }\end{array}$ & $\mid \begin{array}{l}\infty \\
0 \\
0 \\
0 \\
0\end{array}$ & 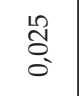 & \begin{tabular}{l}
\multirow{H}{*}{} \\
ᄋ
\end{tabular} & 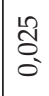 & $\mid \begin{array}{l}\infty \\
\vdots \\
0 \\
0\end{array}$ & $\begin{array}{l}m \\
0 \\
0 \\
0\end{array}$ & 感 & స్ & $\begin{array}{l}\hat{\sigma} \\
\tilde{\sigma} \\
0\end{array}$ & $\begin{array}{l}\hat{a} \\
\vdots \\
0\end{array}$ & \begin{tabular}{|l|l}
10 \\
0 \\
0 \\
0
\end{tabular} & $\begin{array}{l}\vec{H} \\
0 \\
0\end{array}$ & $\frac{m}{a}$ \\
\hline & 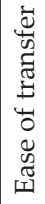 & 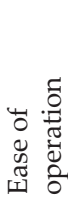 & 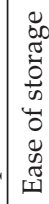 & 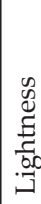 & 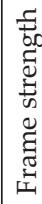 & $\begin{array}{l}0 \\
0 \\
0 \\
0\end{array}$ & 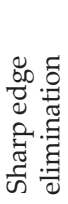 & 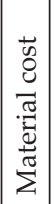 & 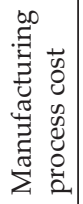 & 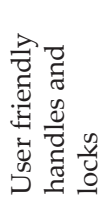 & 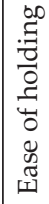 & 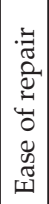 & 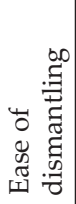 & Ư⿱ & U⿱士口: & $\bigcup_{1}^{3}$ & 茪 & 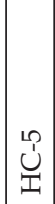 & 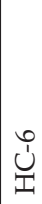 & \\
\hline
\end{tabular}


Table 12. The relative importance of nodes and clusters

\begin{tabular}{|c|c|c|c|c|}
\hline Clusters & Nodes & $\begin{array}{c}\text { (1) Ratio scale priority in the } \\
\text { network }\end{array}$ & $\begin{array}{c}\text { (2) Ratio scale priority of } \\
\text { clusters }\end{array}$ & $\begin{array}{c}(3)=(1) /(2) \text { Ratio scale priority } \\
\text { of nodes in their cluster }\end{array}$ \\
\hline \multirow{5}{*}{ Performance } & Ease of transfer & 0,083 & \multirow{5}{*}{0,306} & 0,271 \\
\hline & $\begin{array}{l}\text { Ease of } \\
\text { operation }\end{array}$ & 0,075 & & 0,245 \\
\hline & Ease of storage & 0,061 & & 0,199 \\
\hline & Lightness & 0,046 & & 0,150 \\
\hline & Frame strength & 0,041 & & 0,134 \\
\hline \multirow[b]{2}{*}{ Safety } & Stability & 0,301 & \multirow[b]{2}{*}{0,41} & 0,734 \\
\hline & $\begin{array}{l}\text { Sharp edge } \\
\text { elimination }\end{array}$ & 0,109 & & 0,266 \\
\hline \multirow[b]{2}{*}{ Cost } & Material cost & 0,038 & \multirow[b]{2}{*}{0,063} & 0,603 \\
\hline & $\begin{array}{l}\text { Manufacturing } \\
\text { process cost }\end{array}$ & 0,025 & & 0,397 \\
\hline \multirow[t]{2}{*}{ Human factors } & $\begin{array}{l}\text { User friendly } \\
\text { handles and } \\
\text { locks }\end{array}$ & 0,044 & \multirow[t]{2}{*}{0,069} & 0,637 \\
\hline & Ease of holding & 0,025 & & 0,362 \\
\hline \multirow[b]{2}{*}{ Maintenance } & Ease of repair & 0,018 & \multirow[b]{2}{*}{0,031} & 0,581 \\
\hline & $\begin{array}{l}\begin{array}{l}\text { Ease of } \\
\text { dismantling }\end{array} \\
\end{array}$ & 0,013 & & 0,42 \\
\hline \multirow{7}{*}{ Alternatives } & $\mathrm{HC}-1$ & 0,024 & \multirow{7}{*}{0,124} & 0,194 \\
\hline & HC-2 & 0,021 & & 0,169 \\
\hline & HC-3 & 0,019 & & 0,153 \\
\hline & HC-4 & 0,018 & & 0,145 \\
\hline & HC-5 & 0,015 & & 0,121 \\
\hline & HC-6 & 0,014 & & 0,113 \\
\hline & HC-7 & 0,013 & & 0,105 \\
\hline
\end{tabular}

As seen in Table 13, the overall performance levels of HCs-1, 2 and 3 are in a descending order in both methods. HC-4 took the first and HC-1 took the second high performance score in the AHP results. The scores they received are close to each other, placing $\mathrm{HC}-1$ also close to the top of the list. In the ANP results, HC-1 took the first place and HC-4 took the fourth place with considerable quantitative score difference. The performance levels of HCs-5 and 6 also follow each other with small difference in performance scores, which also validate the results of both methods. On the other hand, while the place of HC-7 in the list changes according to the two methods, the design concepts HC-5, HC-6, HC-7 have very close performance scores.

As the last step of the research, a validation survey was conducted with the participating professor as a design expert to assess the results obtained from both methods. Similar to the validation procedure performed by Harputlugil et al. (2014), the questions were scored using a 1-10 scale shown in Table 14. During the survey, it was argued that HC-1, with its sophisticated design including a lockable cover, strong sides enabling it to more safely house its contents, ability to be carried like a backpack, being pulled on wheels or carried by handle, and ability to stand by itself when stationary, is a design with higher capability in fulfilling the requirements

\begin{tabular}{|c|c|c|c|c|}
\hline & \multicolumn{2}{|c|}{ ANP } & \multicolumn{2}{c|}{ AHP } \\
\hline $\mathbf{1}$ & HC-1 & 0,194 & HC-4 & 0,161 \\
\hline $\mathbf{2}$ & HC-2 & 0,169 & HC-1 & 0,16 \\
\hline $\mathbf{3}$ & HC-3 & 0,153 & HC-2 & 0,147 \\
\hline $\mathbf{4}$ & HC-4 & 0,145 & HC-3 & 0,14 \\
\hline $\mathbf{5}$ & HC-5 & 0,121 & HC-7 & 0,139 \\
\hline $\mathbf{6}$ & HC-6 & 0,113 & HC-5 & 0,134 \\
\hline $\mathbf{7}$ & HC-7 & 0,105 & HC-6 & 0,119 \\
\hline
\end{tabular}




\begin{tabular}{|c|c|c|}
\hline & Questions (Translated from Turkish) & Scores \\
\hline 1 & Please score the AHP evaluation method for how well its results reflect your personal opinions. & 7 \\
\hline 2 & Please score the ANP evaluation method for how well its results reflect your personal opinions. & 9 \\
\hline 3 & Please score the reliability of the AHP evaluation results. & 7 \\
\hline 4 & Please score the reliability of the ANP evaluation results. & 9 \\
\hline 5 & Please score the AHP evaluation method for its applicability to industrial design. & 9 \\
\hline 6 & Please score the ANP evaluation method for its applicability to industrial design. & 8 \\
\hline 7 & $\begin{array}{l}\text { Please score your level of recommendation for using the AHP method in evaluation tasks at the } \\
\text { industrial design process. }\end{array}$ & 8 \\
\hline 8 & $\begin{array}{l}\text { Please score your level of recommendation for using the ANP method in evaluation tasks at the } \\
\text { industrial design process. }\end{array}$ & 9 \\
\hline
\end{tabular}

Table 14. Survey questions and received scores of the evaluation criteria compared to HC-4. Therefore it was reasoned that the ANP method with its additional capabilities in calculating internal interactions between criteria and alternatives have determined the scores of HC-1 and HC-4 more accurately. According to the validation survey results, ANP received a higher score in reflecting personal opinions and was found to be more reliable. Whereas AHP received a higher score for applicability to industrial design, it was ANP that received a higher score for recommendation to be used in the industrial design process. Finally both methods with their balancing advantages and disadvantages showed similar overall performances in this validation, receiving close scores.

\section{CONCLUSION}

In summary, this article has presented a methodology to facilitate informed design decisions under uncertainty, building on the multi attribute decision-making techniques of AHP and ANP. The use of the AHP and ANP processes as multiple criteria evaluation and decision making tools are investigated for concept evaluation and selection during the industrial design process. Both methods are demonstrated on separate industrial design applications in which the same set of hand cart design concepts are evaluated and the most suitable one is selected for further design development.

The methodology is divided into three segments, each consisting of multiple steps. The first segment involves setting up the problem by defining objectives, priorities, design attributes, and design concept alternatives. The second segment involves data collection from participators. And the last segment is the execution of PC programs to do the necessary calculations for generating the performance scores of the design alternatives.

The contributions of this article fall into three categories. Firstly, AHP and ANP were introduced and comparatively outlined. Secondly, several ways of visualizing and analyzing these methods' results were introduced that will be useful for clearly understanding and interpreting the findings. Thirdly, the proposed decision support process was demonstrated on a practical industrial design application.

By comparing the two sets of results of the evaluation processes and considering participator opinions gathered in a later validation survey, it is concluded that although its application was more complex and time consuming than AHP, ANP gave more accurate results for evaluating design concepts. This is due to ANP's capability of calculating interior relations between the criteria and alternatives. It is found that both AHP 
and ANP with their own advantages are useful for providing a framework for facilitating design decisions under uncertainty. It is concluded that these two methods are only meant to inform, not make a decision, which is the ultimate responsibility of the decision maker himself. Thus, AHP and ANP are most useful in scenarios where large numbers of alternatives need to be reduced to a manageable few, or where it yields a statistically significant 'best' design subject to little dispute. In instances where two or more designs receive close scores, further checks such as the validation survey we used for final testing the results might be necessary. Therefore our study might be considered as an initial guide to generate better and more developed adaptations of the methods to industrial design. In closing, based on the insights of this paper, it is hoped that the methods and ideas presented here are further developed and find broader use with industrial designers in the future.

\section{BIBLIOGRAPHY}

AN, S.H., KIMB, G.H., KANG, K.I. (2007) A Case-Based Reasoning Cost Estimating Model Using Experience by Analytic Hierarchy Process, Building and Environment 42(7) 2573-9.

ANP SOLVER (2016) is a software tool free for academic use that implements the Analytic Network Process developed by Thomas Saaty in 1996 [http://kkir.simor.ntua.gr/anpsolver.html] Access Date (18.08.2018).

AYAĞ, Z. (2005) An Integrated Approach to Evaluating Conceptual Design Alternatives in a New Product Development Environment, International Journal of Production Research 43(4) 687-713.

AZIZI, A., MALEKI, R. (2014) Comparative Study of AHP and ANP on Multi-Automotive Suppliers with Multi-Criteria, Proceedings of the International Conference of Engineers and Computer Scientists 2014 Vol II, IMECS 2014, (March 12 - 14, 2014), Hong Kong [http://www. iaeng.org/publication/IMECS2014/IMECS2014_pp1031-1036.pdf] Access Date (18.08.2018).

BAŞAK, I. (2002) On the Use of Information Criteria in Analytic Hierarchy Process, European Journal of Operational Research 141(1) 200-16.

BATTISTONI, E., COLLADON, A. F., SCARABOTTI, L., SCHIRALDI M. (2013) Analytic Hierarchy Process for New Product Development, International Journal of Engineering Business Management 5(42) 1-8

BAYAZIT, O. (2006) Use of Analytic Network Process in Vendor Selection Decisions, Benchmarking: An International Journal 13(5) 566-79.

BAYRAKÇI, O. (2004) Zihinsel Sınıflandırma Stratejisinin Ürün Tasarımında Örneklenmesi, Çağdaş İletişim Kuramları Açısından Tasarımda İletişimsel Modeller, Mimar Sinan Üniversitesi Yayınları, İstanbul; 130-138.

BEDESSI, S., LISI, S. (2011) Ahp, Anp, And Ann: Technical Differences, Conceptual Connections, Hybrid Models, Proceedings of the International Symposium on the Analytic Hierarchy Process, (2011, Sorrento Italy Editor Emilio Esposito).

BEGIČEVIĆ, N., DIVJAK, B., HUNJAK, T. (2007) Comparison Between Ahp And Anp: Case Study Of Strategic Planning Of E-Learning Implementation, ISAHP 2007, (August 3-8 2007) Viña Del Mar, 
Chile, [http://www.isahp.org/2007Proceedings/Papers/Working\%20 Sessions/Math\%20Subjects/e-learning\%20Implementation\%20 AHP\%20versus\%20ANP.pdf] Access Date (18.08.2018).

BELTRÁN, P.A., GONZÁLEZ, F.C., PASCUAL, J., FERRANDO, P., RUBIO, A.P. (2014) An AHP (Analytic Hi-erarchy Process)/ANP (Analytic Network Process)-Based Multi-Criteria Decision Approach for the Selection of Solar-Thermal Power Plant Investment Projects, Energy, (66)222-38.

BOONKANIT, P., APHIKAJORNSIN, A. (2009) An Energetic Eco-Design at Conceptual Design Phase, International Journal of Industrial Engineering 17(3) 190-9.

BRAUNSCHWEIG, T., BECKER, B. (2004) Choosing Research Priorities by Using the Analytic Hierarchy Process: An Application to International Agriculture, RED Management 34(1) 77-86.

BÜYÜKYAZICI, M., SUCU, M. (2003) The Analytic Hierarchy and Analytic Network Processes, Hacettepe Journal of Mathematics and Statistics (32) 65-73.

CAO, D., LEUNG, L., LAW, J. (2008) Modifying Inconsistent Comparison Matrix in Analytic Hierarchy Process: A Heuristic Approach, Decision Support Systems 44(4) 944-53.

CHANDRAN, B., GOLDEN, B., WASIL, E. (2005) Linear Programming Models for Estimating Weights in the Analytic Hierarchy Process, Computers and Operations Research 32(9) 2235-54.

CHENG E. W. L., LI H. (2004) Contractor Selection Using the Analytic Network Process, Construction Management and Economics (22) 102132.

CHENG, S.C., CHOU, T.C., YANG, C.L., CHANG, H.Y. (2005) A Semantic Learning for Content-Based Image Retrieval Using Analytical Hierarchy Process, Expert Systems with Applications (28) 495-505.

CHOU, Y., HSU, Y.Y. (2008) Human Resources for Science and Technology: Analyzing Competitiveness Using the Analytic Hierarchy Process, Technology in Society 30(2) 141-53.

DAĞDEVİREN, M., YÜKSEL, I. (2007) Personnel Selection Using Analytic Network Process, İstanbul Ticaret Üniversitesi Fen Bilimleri Dergisi 11(6) 99-118.

DAĞDEVİREN, M., DÖNMEZ, N., KURT, M. (2006) Bir İşletmede Tedarikçi Değerlendirme Süreci İçin Yeni Bir Model Tasarımı ve Uygulaması, Gazi Üniversitesi Mühendislik ve Mimarlık Fakültesi Dergisi 21(2) 247-55.

DELİCE, E. K., GÜNGÖR, Z. (2009) The Usability Analysis with Heuristic Evaluation and Analytic Hierarchy Process, International Journal of Industrial Ergonomics 39(6) 934-9.

DONEGAN, H.A., DODD, F.J., MCMASTER, T.B.M. (1992) A New Approach to AHP Decision Making, The Statistician 41(3) 295-302.

ESHTEHARDIAN, E., GHODOUSI, P., BEJANPOUR, A. (2012) Using ANP and AHP for the Supplier Selection in the Construction and Civil Engineering Companies; Case Study of Iranian Company, KSCE Journal of Civil Engineering 17(2) 262-70. 
EXPERT CHOICE COMPARION (2016) Collaborative Solutions for Organizational Decision Making, [http://expertchoice.com/ comparion/] Access Date (19.08.2018).

FELICE, F. D., PETRILLO, A. (2010) A Multiple Choice Decision Analysis: An Integrated QFD - AHP Model for the Assessment of Customer Needs, International Journal of Engineering, Science and Technology 2(9) 25-38.

FUNG, RYK., CHEN, Y., TANG, J. (2007) A Quality-Engineering-Based Approach for Conceptual Product Design. Advanced Manufacturing Technology (32) 1064-73.

GARCIA-CASCALES, M.S., LAMATA, M. (2009) Selection of a Cleaning System for Engine Maintenance Based on the Analytic Hierarchy Process, Computers and Industrial Engineering 56(4) 1442-51.

GODWIN, G. U. (2000) Using Analytic Hierarchy Process to Analyze the Information Technology Outsourcing Decision, Industrial Management and Data Systems 100(9) 421-9.

GRAHAM, A. M. (2012) Using an AHP/ANP Hybrid Methodology for Freight Transport Networks Selection Towards Sustainable Transportation, Unpublished PhD Dissertation, The University of Texas, Arlington.

GUPTA, S., SINGH, S.K., AGRAWAL, K., NAGARAJU, D. (2015) Comparison of Fuzzy ANP and TOPSIS Evaluations of Luggage Bag Design Alternatives, ARPN Journal of Engineering and Applied Sciences 10(14) 6072-9

GÜNGÖR, İ., İŞLER, D. B. (2005) Analitik Hiyerarşi Yaklaşımı İle Otomobil Seçimi, Zonguldak Karaelmas Üniversitesi Sosyal Bilimler Dergisi 1(2) 21-33.

HAFEEZ, K., MALAK, N., ZHANG, Y. (2007) Outsourcing Non-Core Assets and Competences of A Firm Using Analytic Hierarchy Process, Computers and Operations Research 34(12) 3592-608.

HALSKOV, K., HANSEN, N. B. (2015) The Diversity of Participatory Design Research Practice at PDC 2002-2012, International Journal of Human-Computer Studies 74 (February) 81-92.

HAMBALI, A., SALIT, M.S., ISMAIL, N., NUKMAN, Y. (2008) Use of Analytical Hierarchy Process (AHP) for Selecting the Best Design Concept, Jurnal Teknologi 49(A) 1-18.

HAMBALI, A., SALIT, M.S., ISMAIL, N., NUKMAN, Y. (2009) Application of Analytical Hierarchy Process in the Design Concept Selection of Automotive Composite Bumper Beam During the Conceptual Design Stage,Scientific Research and Essay 4(4) 198-211.

HARPUTLUGIIL, T., GÜLTEKİN, A.T., PRINS, M., TOPÇU, Y. İ. (2014) Architectural Design Quality Assessment Based on Analytic Hierarchy Process: A Case Study, METU JFA 31(2) 139-61

HSIAO, S.W. (2002) Concurrent Design Method for Developing a New Product, International Journal of Industrial Ergonomics (29) 41-55.

HSU, W., WOON, I.M.Y. (1998) Current Research in the Conceptual Design of Mechanical Products, Computer-Aided Design (30) 377-89. 
HURLEY, J.W. (2001) The Analytic Hierarchy Process: A Note on an Approach to Sensitivity Which Preserves Rank Order, Computers and Operations Research 28(2) 185-8.

JIAN-ZHONG, X., LI-JING, W., JUN, L. (2008) A Study of AHP-Fuzzy Comprehensive Evaluation on the Development of Eco-Enterprise, International Conference on Management Science and Engineering (10-12 Sept. 2008 Editor Lan HUA) 10(12) 219-24. [http://ieeexplore. ieee.org/document/4668919/?arnumber=4668919\&tag=1] Access Date (19.08.2018).

LEE, D., HWANG, J. (2010) Decision Support for Selecting Exportable Nuclear Technology Using the Analytic Hierarchy Process: A Korean Case, Energy Policy 38(1) 161-7.

KARAGIANNIDIS, A., PAPAGEORGIOU, A., PERKOULIDIS, G., SANIDA, G., SAMARAS, P. (2010) A Multi-Criteria Assessment of Scenarios on Thermal Processing of Infectious Hospital Wastes: A Case Study for Central Macedonia, Waste Management 30(2) 251-62.

KARSAK, E.E., SÖZER, S., ALPTEKIN, S.E. (2002) Product Planning in Quality Function Deployment Using a Combined Analytic Network Process and Goal Programming Approach, Computers and Industrial Engineering 44(1) 171-90.

KIM, K. M., LEE, K. P. (2010) Two Types of Design Approaches Regarding Industrial Design and Engineering Design in Product Design, International Design Conference - Design Dubrovnik - Croatia 1720 May 2010, Editor Dorian Marjanović) [http://dpl.kaist.ac.kr/ wp-content/uploads/DPL_IntConference_18.pdf] Access Date (19.08.2018).

KOÇAK A. (2003) Yazılım Seçiminde Analitik Hiyerarşi Yöntemi Yaklaşımı ve Bir Uygulama, Online Dergi 3(1-2) 67-77.

KURUÜZÜM A., ATSAN N. (2001) Analitik Hiyerarşi, Yöntemi ve İşletmecilik Alanındaki Uygulamaları, Akdeniz İ.İ.B.F. Dergisi (1) 83105.

KWONG, C.K. BAI, H. (2003) Determining the Importance Weights for the Customer Requirements in QFD Using a Fuzzy AHP with an Extent Analysis Approach, IIE Transactions 35(7) 619-26,

LAI V. S., TRUEBLOOD R. P., WONG B. K. (1999) Software Selection: A Case Study of the Application of the Analytical Hierarchy Process to the Selection of Multimedia Authoring System, Information and Management (36) 221-32.

MARINI, C. D., FATCHURROHMAN, N., AZHARI, A., SURAYA, S. (2016) Product Development Using QFD, MCDM and the Combination of These Two Methods, IOP Conference Series 1: Materials Science and Engineering (114) 1-8 (12-14th November 2015, Editor Zamzuri Hamedon)

MIN H. (1994) Location Analysis of International Consolidation Terminals Using the Analytical Hierarchy Process, Journal of Business Logistics 15(2) 25-44.

MYERS, J. H., ALPERT, M. I. (1968) Determinant Buying Attitudes: Meaning and Measurement, Journal of Marketing, 32(4) 13-20. 
NAGAHANUMAIAH R. B., MUKHERJEE, N.P. (2007) Rapid Tooling Manufacturability Evaluation Using Fuzzy-AHP Methodology, The International Journal of Production Research 45(5) 1161-81.

NOVA, N. (2014) Beyond Design Ethnography: How Designers Practice Ethnographic Research, SHS Publishing, France.

ÖZDEN Ü. H. (2008) Analitik Hiyerarşi Yöntemi ile İlkokul Seçimi, Marmara Üniversitesi İ.İ.B.F. Dergisi, 24(1) 299-320.

PENG, X., DAI, F. (2009) Information Systems Risk Evaluation Based on the AHP-fuzzy Algorithm, International Conference on Networking and Digital Society, (30-31May Guizhou/China):178-80 [http://ieeexplore. ieee.org/document/5116713/?arnumber=5116713\&tag=1/] Access Date (19.08.2018).

PINEDA-HENSON R., CULABA A.B., MENDOZA G.A. (2008) Evaluating Environmental Performance of Pulp and Paper Manufacturing Using the Analytic Hierarchy Process and Life-Cycle Assessment, Journal of Industrial Ecology 6(1) 15-28.

RAHARJO, H., BROMBACHER, A.C., XIE, M. (2008) Dealing with Subjectivity in Early Product Design Phase: A Systematic Approach to Exploit Quality Function Deployment Potentials, Computers and Industrial Engineering, 55(1) 253-78,

RAMADHAN, R., AL-ABDUL, V.H., DUFFUAA, S. (1999) The Use of an Analytical Hierarchy Process in Pavement Maintenance Priority Ranking, Journal of Quality in Maintenance Engineering 5(1) 25-39.

RENZI, C., FRANCESCO, L., LUCA, D. A. (2017) A Review on DecisionMaking Methods in Engineering Design for the Automotive Industry, Journal of Engineering Design 28(2) 1-26.

SAATY, T. L. (1980) The Analytic Hierarchy Process, McGraw Hill International Book Company, New York.

SAATY T. (1986) Axiomatic Foundations of the AHP, Management Science 32(7) $841-55$

SAATY, T.L., ÖZDEMIR, S.M. (2003) Why the Magic Number Seven Plus or Minus Two, Mathematical and Computer Modeling 38(3-4) 233-44.

SAATY T.L. (2004) Decision making - the Analytic Hierarchy and Network Processes (AHP/ANP), Journal of Systems Science and Systems Engineering 13(1) 1-35.

SAATY, T.L. (2008) Decision Making with the Analytic Hierarchy Process, International Journal of Services Sciences 1(1) 83-98.

SAATY, T.L., SHIH, H.S. (2009) Structures in Decision Making: On the Subjective Geometry of Hierarchies and Networks, European Journal of Operational Research 199(3) 867-72.

SALONEN, M, PERTTULA, M. (2005) Utilization of Concept Selection Methods - A Survey of Finnish Industry. In Proceeding of Engineering Technical Conferences and Computers and Information in Engineering Conference (24-28 September, 2005), Long Beach, California, USA. [http://proceedings.asmedigitalcollection.asme.org/ proceeding.aspx?articleid=1572197] Access Date (20.08.2018). 
SARFARAZ, A., JENAB, K. (2012) A Fuzzy Conceptual Design Selection Model Considering Conflict Resolu-tion, IACSIT International Journal of Engineering and Technology 4(1)38-45.

SHARMA, A., GUPTA, P., SRIVASTAVA, R. K. (2015) Application of AHP and ANP Methods for Selection of Best Material for an Axle, International Journal of Innovative Research in Science, Engineering and Technology 4(5) 2894-901.

SHARMA, M.J., MOON, I., BAE H. (2008) Analytic Hierarchy Process to Assess and Optimize Distribution Network, Applied Mathematics and Computation 202(1) 256-65.

SİPAHI, S., TIMOR, M. (2010) The Analytic Hierarchy Process and Analytic Network Process: An Overview of Applications, Management Decision 48(5) 775-808.

STAIN W.E., MIZZI, P.J. (2007) The Harmonic Consistency Index for the Analytic Hierarchy Process, European Journal of Operational Research 177(1) 488-97.

TOKSARI, M., TOKSARI, D. (2011) Bulanık Analitik Hiyerarşi Prosesi (AHP) Yaklaşımı Kullanılarak Hedef Pazarın Belirlenmesi", ODTU Gelişme Dergisi 38 (Nisan) 51-70.

ULRICH, K. T., EPPINGER, S. D. (2008) Product Design and Development, McGraw-Hill, Singapore.

VAIDYA, O. S., KUMAR, S. (2006) Analytical Hierarchy Process: An Overview of Applications, European Journal of Operational Research (169) 1-29.

WANG, Y., LIU, J. ELHAG, T. (2008) An Integrated AHP-DEA Methodology for Bridge Risk Assessment, Computers and Industrial Engineering 54(3) 513-25.

WEY, W., CHIU, Y. (2013) Assessing the Walkability of Pedestrian Environment Under the Transit-Oriented Development, Habitat International (38) 106-18.

WIND, Y., SAATY, T. L. (1980) Marketing Applications of the Analytic Hierarchy Process, Management Science, 26(7) 641-745

XIAO, A., PARK, S.S., FREIHEIT, T. (2007) A Comparison of Concept Selection in Concept Scoring and Axiomatic Design Methods, Proceedings of the Canadian Engineering Education Association Conference (22-24July 2007, Winnipeg, Manitoba). [https://ojs. library.queensu.ca/index.php/PCEEA/article/view/3769] Access Date (20.08.2018).

XU, L., LI, Z., SHANCANG, L., FENGMING T. (2007) A Decision Support System for Product Design in Concurrent Engineering, Journal of Decision Support Systems 42(4) 2029-42.

YILMAZ, E. (1999) Analitik Hiyerarşi Süreci Kullanılarak Çok Kriterli Karar Verme Problemlerinin Çözümü, DOA Dergisi (5) 95-122.

YILMAZ, M. (2010) Analitik Hiyerarşi Süreci (AHS) ve Bir Uygulama: Lider Bir Kütüphane Müdürü Seçimi, Türk Kütüphaneciliği 24(2) 20634.

ZAHEDI, F. (1986) The Analytical Hierarchy Process - A Survey of the Method and Its Applications, Interfaces 16(4) 96-108. 
ZHANG, C., JIANG, Z., LU, D., REN, T. (2006) 3D MEMS Design Method Via Solidworks Proceeding of the 1st IEEE International Conference on Nano/Micro Engineered and Molecular Systems,(18-21 January 2006, Zhuhai/China) 747-751, [http://diyhpl.us/ nmz787/ pdf/3D_MEMS_Design_Method_via_SolidWorks.pdf] Access Date (20.08.2018).

ZHOU, Y.D., SHI, M.L. (2009) Rail Transit Project Risk Evaluation Based on AHP Model, Second International Conference on Information and Computing Science (May 21-22, 2009, Manchester/UK) (3), 236-8. [https://www.infona.pl/resource/bwmeta1.element.ieeeart-000005168848] Access Date (20.08.2018).

\section{SYMBOLS AND ABBREVIATIONS}

$\lambda$ : Base Value Coefficient

AHP: Analytical Hierarchy Process

AHY: Analitik Hiyerarşi Süreci

ANP: Analytical Network Process

AHN: Analitik Ağ Süreci

CI: Consistency Index

CR: Consistency Ratio

DEA: Data Envelopment Analysis

DFA: Design For Assembly

FMEA: Failure Mode Effects Analysis

GIS: Geographic Information System

HC-n: Hand Cart No n

PC: Personal Computer

PD: Participatory Design

QFD: Quality Function Deployment

RI: Standard Correction Index Value

SWOT: Strengths, Weaknesses, Opportunities and Threats

TOPSIS: Technique for Order Preference by Similarity to Ideal Solution

Alındı: 28.11.2015; Son Metin: 24.05.2018

Anahtar Sözcükler: Analitik hiyerarşi süreci; analitik ağ süreci; tasarım fikri seçimi; ürün tasarımı; pazar çantası tasarımı.

\section{ANALITIKK HİYERARŞİ VE ANALİTIK AĞ SÜREÇLERI KULLANILARAK KAVRAMSAL ÜRÜN TASARIMLARININ DEĞERLENDİRILMESİ}

Bu çalışmanın amacı iki özelleşmiş değerlendirme ve seçim yöntemini endüstri tasarımına uyarlayıp karşılaştırmalı olarak sunmak, böylece endüstri tasarımı sürecinin parçası olan tasarım kavramı değerlendirme işleminin başarı ve verimliliğini artırmaktır. Kullanılacak yöntemler, Analitik Hiyerarşi Süreci (AHP) ve Analitik Ağ Süreci (AHN) olarak adlandırılmaktadır. Yöntemlerin uygulanmasında öncelikle amaç belirlenmekte, sonra değerlendirme ölçütleri ve kavramsal tasarım alternatifleri listelenmektedir. Sonrasında alternatif ve ölçütlerin birbirlerine göre önem dereceleri ve ilişkileri araştırılmaktadır. Sonra bu önem dereceleri ve yöntemlerce sağlanan araçlar kullanılarak tasarım alternatifleri ölçütler açısından değerlendirilmektedir. İki yaklaşımın sınanması için, yedi adet pazar çantası tasarım fikrinin her iki yöntem kullanılarak değerlendirildiği bir uygulama sunulmuştur. İlk olarak 
her iki süreçte ortak olan işlemler açıklanmış, sonra yönteme özel detaylara geçilmiştir. Ortak bölümde, değerlendirmede kullanılacak ölçütler belirlenmiş ve bunların ürün başarımına yapması beklenen etki, uzmanlarla gerçekleştirilen görüşmelerde yapılan ikili karşılaştırmalarla bulunmuştur. Sonrasında eldeki yedi pazar çantası tasarım alternatifi, ölçütler ve ilişkiler açısından her iki yöntem kullanılarak değerlendirilmiş ve göreceli başarım değerleri ortaya çıkartılmıştır. Elde edilen sonuçlar, büyükten küçüğe sıralanmış ve en yüksek göreceli önem değerine sahip tasarım, en başarılı ürün kavram tasarımı olarak belirlenmiştir. İki yöntemden alınan sonuçlar karşılaştırmalı olarak yorumlanmıştır.

\section{PRODUCT DESIGN CONCEPT EVALUATION BY USING ANALYTICAL HIERARCHY AND ANALYTICAL NETWORK PROCESSES}

The aim of this study is to improve the success and efficiency of the design concept evaluation and selection activity in industrial design by adapting two specialized evaluation-selection methods to the design process and comparatively demonstrating their use. The methods are called the Analytic Hierarchy Process (AHP) and the Analytic Network Process (ANP). In their application, first the main design objective is decided upon and the evaluation criteria are determined accordingly, followed by the listing of the design concept alternatives. Then the criteria's importance values as well as relations between them are investigated and evaluation of the alternatives is done according to the criteria by using the tools provided by the methods. The application presented in the article evaluates seven hand cart designs by using the two methods. First the tasks that are common in both methods, then the tasks specific to each method are explained. The general criteria to be used in the evaluation are identified and the effects of these criteria on the evaluation process are determined through interviews with designers and users participating in the study. Then the relative performance rankings of the available seven hand cart alternatives are calculated by using both methods. Numerical results are listed in a descending order to show the success levels of the design concepts, pointing to the one that received the highest overall performance. The findings obtained from two methods are comparatively interpreted.

HÜSEYIN ÖZKAL ÖZSOY; B.Eng., M.Sc., Ph.D.

Received his bachelor's degree in electronics engineering from İstanbul Technical University in 1993 and his first master's degree in engineering from Boğaziçi University in 1996 and his second master's degree in industrial design from Mimar Sinan Fine Arts University in 2001. Earned his Ph.D. degree in industrial design from Mimar Sinan Fine Arts University in 2009. Major research interests include production oriented computer aided design, industrial design engineering, multidisciplinary research, intelligent products, embedded product software technologies and prototyping. ozkal.ozsoy@msgsu.edu.tr.

ÇİĞDEM YILMAZ ÖZSOY; B.Sc., M.Sc.

Received her B.Sc. of Statistics from Istanbul Commerce University, Faculty of Economics in 2013. Earned her master's degree in econometrics from Istanbul University in 2015. Currently a Ph.D. candidate in Istanbul University, Faculty of Economics. Major research interests include multidisciplinary studies and statistical evaluation-validation techniques of scientific research data. cigdem_yilmazz@hotmail.com 